\title{
USP3 inhibits type I interferon signaling by deubiquitinating RIG-I-like receptors
}

Jun Cui ${ }^{1,2, *}$, Yanxia Song ${ }^{2,3, *}$, Yinyin $\mathrm{Li}^{2,4, *}$, Qingyuan $\mathrm{Zhu}^{2}$, Peng Tan ${ }^{2,4}$, Yunfei Qin ${ }^{1}$, Helen Y Wang ${ }^{2}$, Rong-Fu Wang ${ }^{2}$

${ }^{I}$ Key Laboratory of Gene Engineering of the Ministry of Education, State Key Laboratory of Biocontrol, College of Life Sciences, Sun Yat-sen University, Guangzhou 510275, China; ${ }^{2}$ Center for Inflammation and Epigenetics, The Methodist Hospital Research Institute, Houston, TX 77030, USA; ${ }^{3}$ Central Laboratory, The First Affiliated Hospital, Jilin University, Changchun 130012, China: ${ }^{4}$ Institute of Biosciences and Technology, Texas A\&M University Health Science Center, Houston, TX 77030, USA

Lysine 63 (K63)-linked ubiquitination of RIG-I plays a critical role in the activation of type I interferon pathway, yet the molecular mechanism responsible for its deubiquitination is still poorly understood. Here we report that the deubiquitination enzyme ubiquitin-specific protease 3 (USP3) negatively regulates the activation of type I interferon signaling by targeting RIG-I. Knockdown of USP3 specifically enhanced K63-linked ubiquitination of RIG-I, upregulated the phosphorylation of IRF3 and augmented the production of type I interferon cytokines and antiviral immunity. We further show that there is no interaction between USP3 and RIG-I-like receptors (RLRs) in unstimulated or uninfected cells, but upon viral infection or ligand stimulation, USP3 binds to the caspase activation recruitment domain of RLRs and then cleaves polyubiquitin chains through cooperation of its zinc-finger Ub-binding domain and USP catalytic domains. Mutation analysis reveals that binding of USP3 to polyubiquitin chains on RIG-I is a prerequisite step for its cleavage of polyubiquitin chains. Our findings identify a previously unrecognized role of USP3 in RIG-I activation and provide insights into the mechanisms by which USP3 inhibits RIG-I signaling and antiviral immunity.

Keywords: innate immunity; type I interferon signaling; RIG-I-like receptors; deubiquitinase

Cell Research (2014) 24:400-416. doi:10.1038/cr.2013.170; published online 24 December 2013

\section{Introduction}

The innate immune system serves as the first line of defense against invading viruses by sensing pathogenassociated molecular patterns (PAMPs). Viral RNA and DNA can be recognized by a variety of pattern recognition receptors (PRRs), including Toll-like receptors (TLRs), Nod-like receptors (NLRs), RIG-I-like receptors (RLRs) and several additional sensors of DNA [13]. TLR3, TLR7, TLR8 and TLR9 are important sensors that detect viral DNA or RNA in the endosome and trigger TIR domain-containing adaptor-inducing interferon

\footnotetext{
*These three authors contributed equally to this work.

Correspondence: Jun Cui ${ }^{\mathrm{a}}$, Rong-Fu Wang ${ }^{\mathrm{b}}$

âE-mail: cuij5@mail.sysu.edu.cn

${ }^{b} E$-mail: rwang3@tmhs.org

Received 10 July 2013; revised 15 October 2013; accepted 11 November 2013; published online 24 December 2013
}

(IFN)-beta (TRIF)- and MYD88-mediated signaling pathways $[1,3]$. By contrast, RIG-I and MDA5 (melanoma differentiation-associated gene 5) function as cytoplasmic RNA sensors and recruit the mitochondrial protein called MAVS (mitochondrial antiviral signaling, also known as VISA, IPS-1 or Cardif) after stimulation $[1,2]$. Recent studies show that RNA polymerase III can serve as an intracellular viral DNA sensor by transcribing viral AT-rich double-strand DNA into double-strand RNA (dsRNA), which in turn stimulates RIG-I and initiates the MAVS-dependent signaling cascade [4, 5]. Furthermore, DAI (DNA-dependent activator of IFN-regulatory factors), AIM2 (absent in melanoma 2), IFI16 (IFN-gamma inducible protein 16) and DDX41 (DEAD (Asp-Glu-AlaAsp) box protein 41) function as cytosolic DNA sensors and recruit stimulator of IFN genes (STING) to activate the type I IFN signaling pathway [6-10].

Upon PAMP stimulation, these PRRs trigger the activation of NF- $\mathrm{BB}$, type I IFN and inflammasome signal- 
ing pathways, leading to production of proinflammatory cytokines. Although type I IFN is critically required for viral clearance, aberrant type I IFN production (including IFN- $\alpha$ and IFN- $\beta$ ) can play a role in immunopathology and autoimmune disorders [11]. Thus, tight regulation of type I IFN signaling is critical to maintain homeostasis of both innate and adaptive immunity $[12,13]$. Ubiquitination is an important post-translational modification that plays a critical role in the positive or negative regulation of innate immune signaling pathways [14]. MDA5 shares with RIG-I the same domain architecture and contains two N-terminal tandem caspase activation recruitment domains (CARDs) for binding to MAVS, a central $\mathrm{DExD} / \mathrm{H}$ motif helicase domain responsible for RNA-dependent ATP hydrolysis and a C-terminal domain (CTD) for dsRNA binding [2]. While RIG-I recognizes dsRNA ends containing 5 'ppp and displays a strong preference for shorter $(<1-2 \mathrm{~kb})$ dsRNA, MDA5 recognizes longer $(>$ $2 \mathrm{~kb})$ dsRNA through filament formation along dsRNA $[15,16]$. Recent structural analysis shows that RIG-I is autorepressed in the absence of viral RNAs through intramolecular interactions between CARD and CTD [1719]. Recognition or binding of viral dsRNA ligand by the CTD of RIG-I releases the CARDs from CTD repression, which interacts with MAVS to stimulate the downstream signaling pathway; however, activation of RIG-I requires both RNA binding and K63-linked polyubiquitin chains [20]. Remarkably, the unanchored K63-linked polyubiquitin chains can potently activate RIG-I and promote the conformational changes or aggregates of MAVS for the subsequent activation of downstream signaling $[2,17$, 20]. Several E3 ubiquitin ligases, including TRIM 25 and Riplet/RNF135/REUL, have been identified to mediate the K63-linked ubiquitination of RIG-I [21, 22]. However, very little is known about proteins that are responsible for removing polyubiquitin chains of RLRs to dampen a robust immune response.

The deubiquitination enzyme ubiquitin-specific protease 3 (USP3) has been identified as a chromatin modifier to maintain the genome integrity [23], but its involvement in innate immune signaling is not clear. Here we report that USP3 functions as a negative regulator of the type I IFN signaling pathway by removing polyubiquitin chains of RIG-I. USP3 specifically binds to RIGI through K63-linked polyubiquitin chains and removes them. Knockdown of USP3 markedly enhances RLRmediated, but not TLR- or DNA sensor-mediated, type I IFN responses. Our findings identify previously unrecognized role of USP3 in the control of innate immune signaling by deubiquitinating K63-linked polyubiquitin chains on RIG-I, thus negatively regulating RLR-mediated innate immune response.

\section{Results}

\section{USP3 negatively regulates type I IFN signaling}

To identify the possible deubiquitinase (DUB) that control type I IFN signaling in antiviral immunity, we transfected HEK293T (human embryonic kidney 293T) cells with an active form of RIG-I (RIG-I (N)), an IFN- $\beta$ luciferase reporter and an internal control renilla TK luciferase, as well as one of 81 candidate genes encoding DUBs (including USPs 1-54, ubiquitin C-terminal hydrolases, Otubain proteases, Machado-Joseph disease domain proteases and JAMM domain proteases). Among them, we identified USP3 as a potent negative regulator of type I IFN signaling (Supplementary information, Figure S1A). To determine whether USP3 can inhibit type I IFN signaling activated by RLR ligands or virus infection, we treated the USP3-transfected cells with intracellular low molecular weight (LMW) poly(I:C) (a ligand for RIG-I), intracellular high molecular weight (HMW) poly(I:C) (a ligand for MDA5), poly(dA:dT) or infected with vesicular stomatitis virus tagged with enhanced green fluorescent protein (VSV-eGFP), and showed that USP3 markedly inhibited the activities of the IFN- $\beta$ luciferase reporter induced by different intracellular stimuli (Figure 1A). Since IFN- $\beta$ activation requires the coordinated signaling from both IRF3- and NF- $\kappa$ Bmediated pathways, we used an IFN-stimulated response element (ISRE) luciferase reporter (which requires IRF3mediated signaling only) and $\mathrm{NF}-\kappa \mathrm{B}$ luciferase reporter, separately, to determine whether the inhibition of IFN- $\beta$ activation by USP3 requires the participation of NF- $\kappa B$ signaling. We found that USP3 markedly inhibited ISREluc activation induced by all the stimuli (Figure 1B), but moderately inhibited NF- $\mathrm{kB}$-luc activation (Supplementary information, Figure S1B), suggesting that USP3 directly and predominantly inhibits IFN- $\beta$ signaling. To further determine whether USP3 inhibits type I IFN pathway, we assessed the phosphorylation of IRF3 in 293 T cells expressing Myc-USP3 or native USP3 after treatment with intracellular LMW and HMW ploy(I:C), poly(dA:dT) or infected with VSV-eGFP, and found that both Myc-tagged or untagged USP3 showed similar inhibitory effects on ISRE activity (Supplementary information, Figure S1C). More importantly, Myc-USP3 markedly inhibited the phosphorylation of endogenous IRF3 induced by different stimuli (Figure 1C). However, ectopic expression of USP3 could not inhibit extracellular poly(I:C)-induced TLR3-mediated IFN- $\beta$ or ISRE activation (Supplementary information, Figure S1DS1E), suggesting that USP3 may specifically affect key molecules such as RIG-I and MDA5 upstream of type I IFN signaling. 

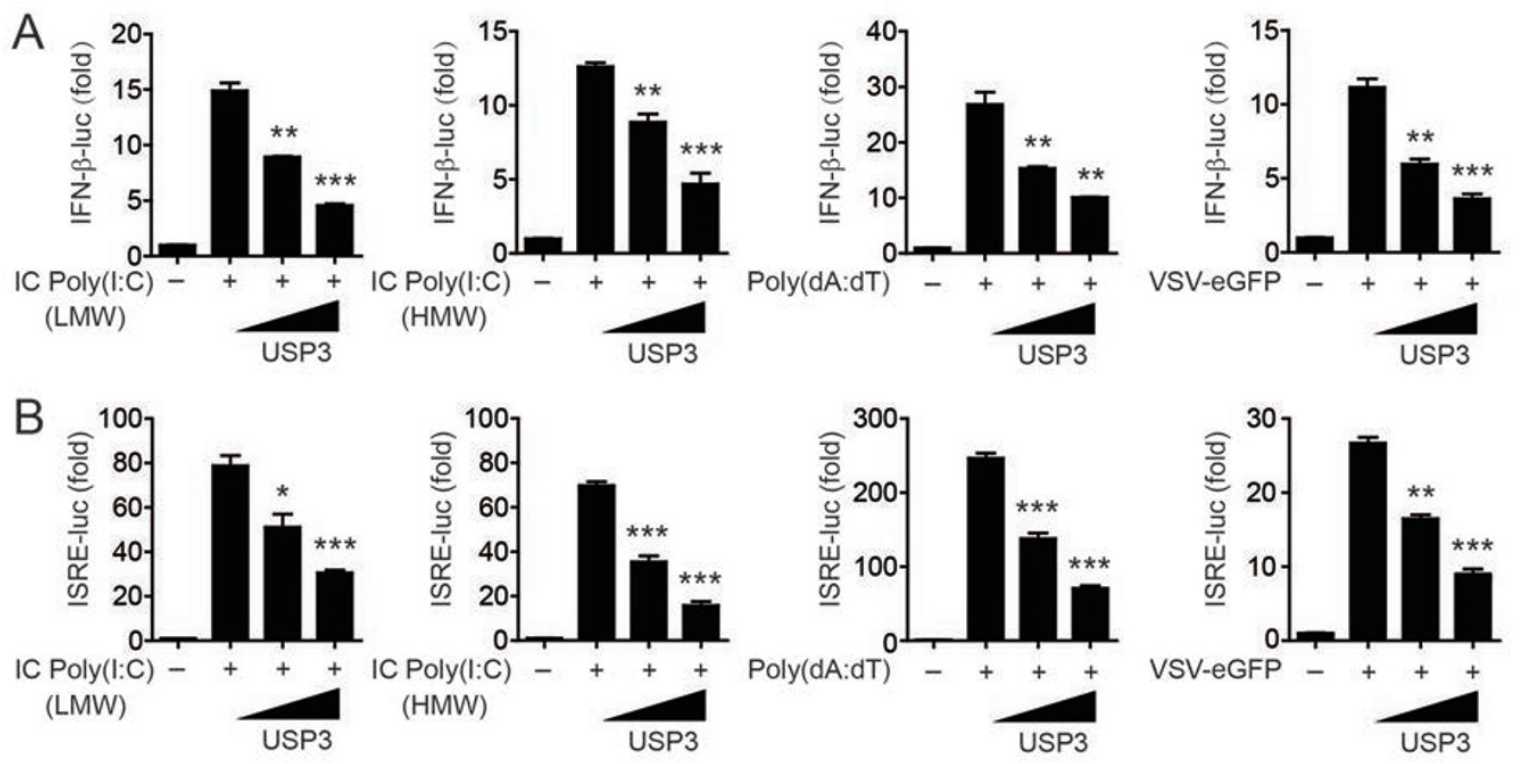

C

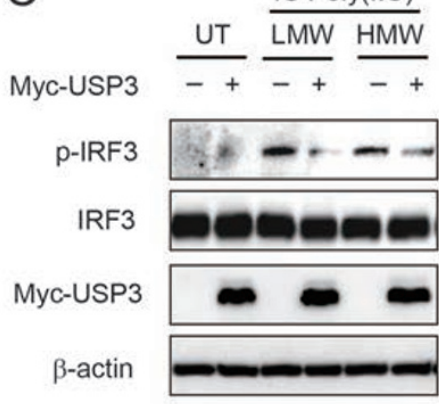

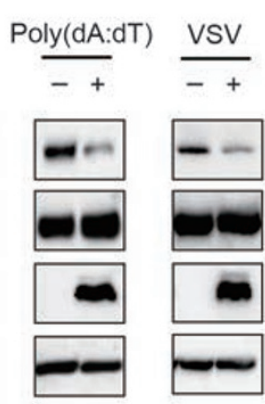

D
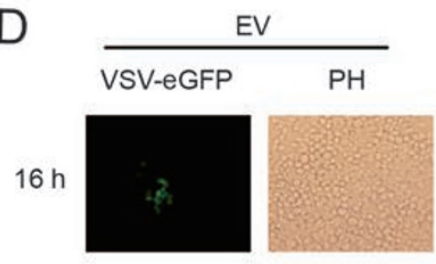

$24 \mathrm{~h}$

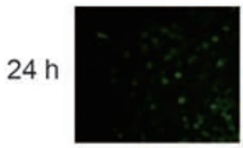

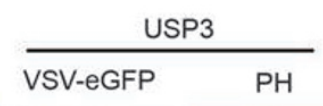

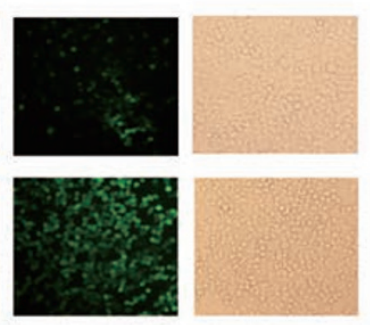

Figure 1 USP3 negatively regulates the type I IFN signaling pathway. (A, B) Luciferase activity in 293T cells transfected with plasmid encoding a luciferase reporter for IFN- $\beta$ (IFN- $\beta$-luc) (A) or ISRE (ISRE-luc; $100 \mathrm{ng}$ each) (B), together with empty vector (no wedge) or an expression vector for USP3 (0, 200 and $400 \mathrm{ng}$; wedge), followed by no treatment or treatment with intracellular (IC) low molecular weight (LMW) poly(l:C) $(1 \mu \mathrm{g} / \mathrm{ml})$, IC high molecular weight (HMW) poly(l:C) $(1 \mu \mathrm{g} / \mathrm{ml})$, poly(dA:dT) $(1 \mu \mathrm{g} / \mathrm{ml})$ or VSV-eGFP $(\mathrm{MOI}=0.1)$. Data are presented relative to Renilla luciferase activity. (C) Immunoblot analysis of total and phosphorylated ( $p$-) IRF3 in 293T cells transfected with empty vector or Myc-tagged USP3, followed by the same treatment set as in A. (D) Phase-contrast (PH) and fluorescence microscopy analyses of 293T cells transfected with empty vector (EV) or an expression vector of USP3, and then infected with VSV-eGFP at an MOI of 0.01 at the indicated time. Original magnification, $\times 10$. Data in $\mathbf{A}, \mathbf{B}$ are presented as the means $\pm \mathrm{SD}$ of three independent experiments. ${ }^{*} P<0.05$, ${ }^{* *} P<0.01$ and ${ }^{* * *} P<0.001$, versus cells with the same treatment without USP3 expression (Student's $t$-test).

To establish a link between reduced type I IFN response mediated by USP3 and antiviral immunity, we cotransfected expression vector of USP3 or empty vector into $293 \mathrm{~T}$ cells, then infected the cells with VSV-eGFP (multiplicities of infection $(\mathrm{MOI})=0.01)$, and monitored viral infection based on GFP expression. Overexpression of USP3 rendered the cells more susceptible to viral infection and resulted in considerably more GFP+ (virusinfected) cells than transfection with empty vector at different times (Figure 1D). Flow cytometry analysis revealed that $30.6 \%$ of cells were infected (GFP+) in cells transfected with empty vector, compared to $87.6 \%$ of GFP+ cells in cells transfected with USP3 $24 \mathrm{~h}$ post infection (Supplementary information, Figure S1F). These results suggested that ectopic expression of USP3 inhibits type I IFN response and thus attenuates antiviral immunity.

\section{Knockdown of USP3 enhances RLR-mediated antiviral responses \\ To determine whether specific knockdown of endog- enous USP3 would increase antiviral responses under physiological conditions, we selected three USP3-spe- cific lentivirus-based short hairpin RNA (shRNA) con-}


structs and an USP3-specific small interfering RNA (siRNA) to knock down the expression of USP3. The USP3 siRNA and two of the three USP 3 shRNAs efficiently inhibited the expression of transfected and endogenous USP3 in 293 T cells and THP-1 cells (Figure 2A and Supplementary information, Figure S2A). We next tested the effect of USP 3 knockdown on the phosphorylation of transcription factor IRF3. As shown in Figure 2B and Supplementary information, Figure S2B-S2C, the phosphorylation of IRF3 (p-IRF3) in the USP3-knockdown cells was at least 3 -fold higher than that in the control shRNA-transfected cells from 6 to $8 \mathrm{~h}$ after intracellular poly(I:C) (LMW or HMW), poly(dA:dT) treatment or the VSV infection, although the total amounts of IRF3 proteins were comparable between USP3-knockdown and control cells. The similar results were obtained when cells were transfected with another USP3 shRNA for knockdown of endogenous USP3 (Supplementary information, Figure S2B). Collectively, these results suggest that specific knockdown of USP3 strongly enhances the phosphorylation of IRF3 after ligand stimulation or viral infection in 293T cells.

We next sought to determine whether the enhanced IRF3 phosphorylation by USP3 knockdown promotes type I IFN activation and type I IFN-dependent gene expression. Using the ISRE and NF- $\mathrm{BB}$ luciferase reporter assay, we showed that knockdown of USP3 markedly increased the ISRE-luc and NF- $\kappa \mathrm{B}-$ luc activity induced by intracellular poly(I:C) (LMW or HMW), poly(dA:dT) or infection with VSV-eGFP in 293T cells (Figure 2C and Supplementary information, Figure S2D). Consistent with this observation, knockdown of USP 3 resulted in markedly increased mRNA abundance of $I F N-\beta$ or IFNstimulated cytokines, such as IFIT2 and CCL5 (Figure 2D). To substantiate these findings, we found that like its human homolog, knockdown of $m U S P 3$ also increased mRNA abundance of IFN- $\beta$ and IFIT2 in mouse macrophage cell line (RAW264.7) by intracellular poly(I:C) treatment or VSV-eGFP infection (Supplementary information, Figure S2H). To further demonstrate the effects of USP 3 knockdown on the secretion of IFN- $\beta$ proteins, we knocked down USP3 in 293T, THP-1 cells, human peripheral blood mononuclear cells (PBMCs) or RAW264.7 cells, and then treated the cells with intracellular poly(I:C) or infected the cells with VSV-eGFP. We found that both poly(I:C) treatment and VSV-eGFP infection resulted in much higher expression of IFN- $\beta$ protein in cells transfected with USP3-specific siRNA (or shRNA) than those transfected with control siRNA (Figure 2E and Supplementary information, Figure S2ES2F, S2I). These results suggest that USP3 knockdown enhances IFN- $\beta$ activation and the expression of IFN- stimulating genes in both human and mouse cells. Interestingly, knockdown of USP3 in THP-1 cells did not affect the IFN- $\beta$ secretion stimulated by Poly(dA:dT) or extracellular Poly(I:C) (a ligand for TLR3) treatment (Supplementary information, Figure S2G), indicating that USP3 has no effect on TLR3- or DNA sensor-mediated type I IFN response.

To further determine whether the enhanced type I IFN response is correlated with antiviral immunity, we knocked down USP3 expression in 293 cells and then infected the cells with VSV-eGFP $(\mathrm{MOI}=0.05)$. Knockdown of USP3 rendered the cells resistant to viral infection and resulted in considerably fewer GFP+ (virusinfected) cells than treatment with control siRNA from 16 to $24 \mathrm{~h}$ post infection (Figure 2F). Flow cytometry analysis revealed that $20 \%$ cells were infected (GFP+) in cells transfected with USP3-specific siRNA, compared to $53 \%$ of GFP+ cells in cells transfected with control siRNA $16 \mathrm{~h}$ post infection (Figure 2G). Knockdown of mUSP3 in RAW264.7 cells shows the similar pattern (Supplementary information, Figure S2J). Taken together, these results suggest that USP3-specific knockdown markedly enhances the type I IFN response and antiviral immunity.

\section{USP3 inhibits type I IFN signaling by targeting RIG-I and MDA5}

As USP3 specifically inhibits type I IFN signaling, we next sought to determine whether USP3 directly modulates the function of RIG-I or MDA5. 293T cells were transfected with $R I G-I$ or MDA5, together with increasing amounts of USP 3 plus the ISRE luciferase reporter, and then treated with intracellular poly(I:C). We found that USP3 markedly inhibited RIG-I- or MDA5mediated ISRE-luc activation after poly(I:C) stimulation (Figure 3A). However, in the absence of ligand stimulation, we observed only a weak activity of ISRE-luc, which was not affected by USP3 expression (Figure 3A), suggesting that USP3 inhibits the activated form of RIGI and MDA5. To test this possibility, we generated Nterminal RIG-I (containing the CARD, RIG-I (N)) or Nterminal MDA5 (containing the CARD, MDA5 $(\mathrm{N})$ ) and then tested their ability to activate ISRE-luc reporter. We found that RIG-I (N) or MDA5 (N) could strongly activate ISRE-luc, but such an activity could be markedly inhibited by increasing amounts of USP3 (Figure 3B), suggesting that USP3 directly inhibits the function of the CARDs of RIG-I and MDA5.

To determine whether USP3 also affects the function of the downstream signaling proteins such as MAVS, STING, TBK1, IRF3 or TIRF, we transfected 293T cells with vectors for MAVS, TRIF, STING, TBK1, or IRF3, 
A

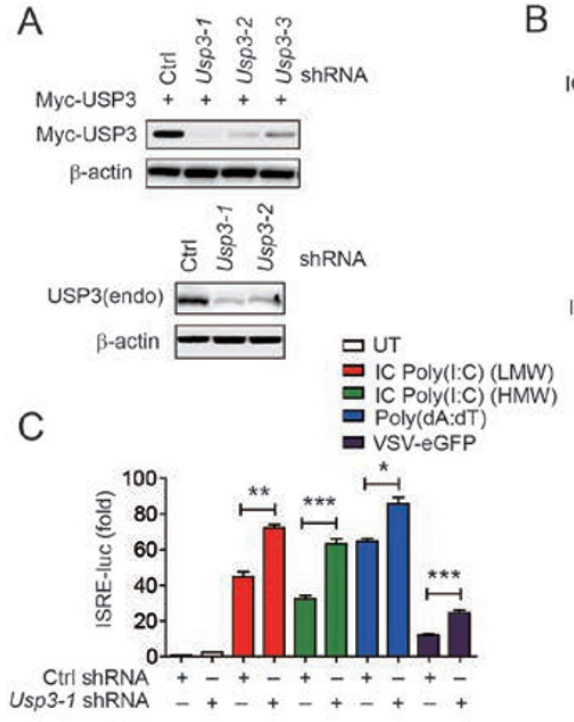

D

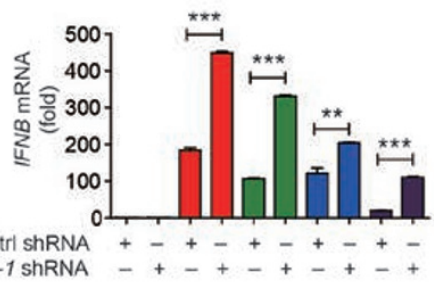

$B$

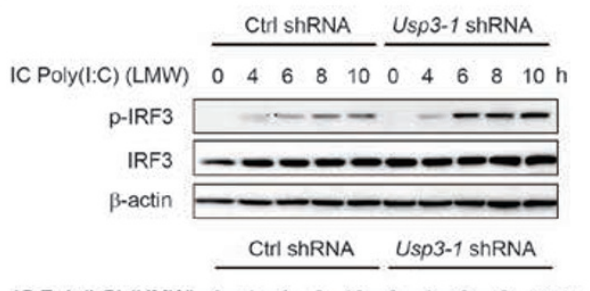

IC Poly(I:C) (HMW) $0 \begin{array}{llllllllll}0 & 4 & 6 & 8 & 10 & 0 & 4 & 6 & 8 & 10\end{array}$

IRF3

$\beta$-actin

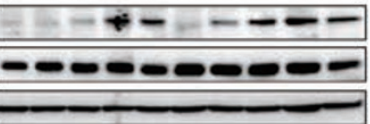

Cirl shRNA Usp3-1 shRNA

$\begin{array}{llllllllllll}\text { VSV-eGFP } & 0 & 4 & 6 & 8 & 10 & 0 & 4 & 6 & 8 & 10 & h\end{array}$

p-IRF3

IRF3

$\beta$-actin
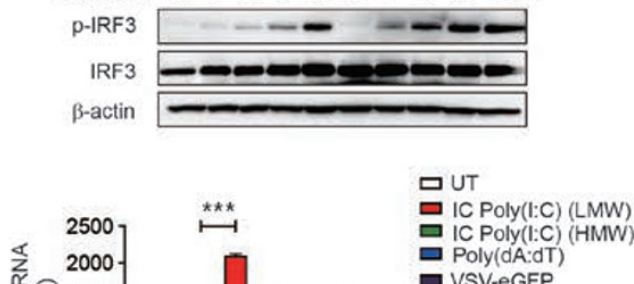

V VSV-eGFP

Ctrl ShRNA $++\ldots+\ldots+\ldots+\ldots+$

Usp3-1 ShRNA $\square$ Ctrl SiRNA

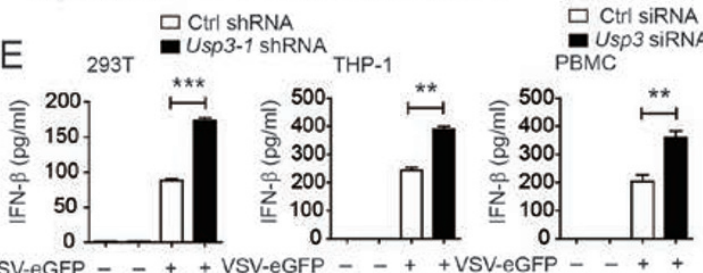

Ctrl shRNA + $++\ldots+\ldots+\ldots+-$ VSV-eGFP -+++ VSV-eGFP -+++ VSV-eGFP -+++

Usp3-1 ShRNA -++-+++++++
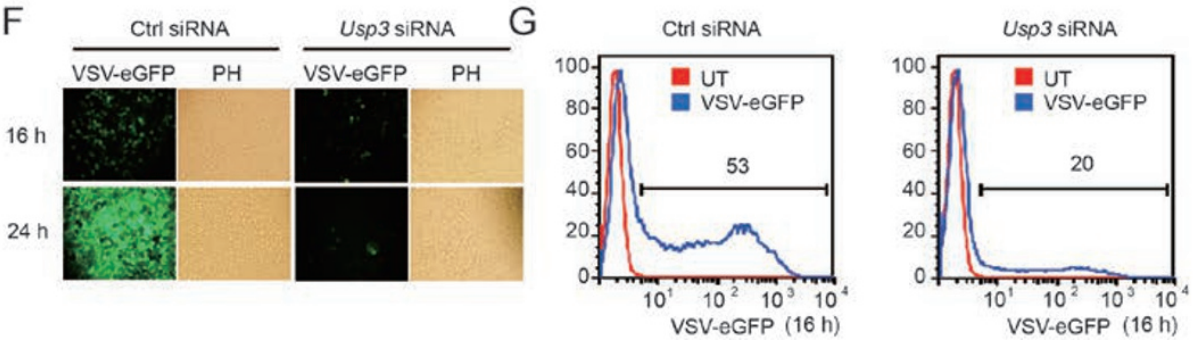

Figure 2 Knockdown of Usp3 enhances IFN- $\beta$ expression as well as antiviral responses. (A) Immunoblot analysis of the knockdown of exogenous USP3 in 293T cells expressing Myc-USP3 (top) or endogenous (endo) USP3 in 293T cells (bottom) treated with Usp3-specific shRNAs or control (Ctrl) shRNA. $\beta$-actin serves as a loading control throughout. (B) Immunoblot analysis of total and phosphorylated (p-) IRF3 in 293T cells transfected with control or Usp3-specific shRNA, followed by treatment with intracellular poly(I:C) (LMW or HMW) or infection with VSV-eGFP at different time points. (C) Luciferase activity in $293 \mathrm{~T}$ cells transfected with Usp3-specific or ctrl shRNA, together with an ISRE luciferase reporter, then left untreated (UT) or treated with intracellular poly(I:C) (LMW), poly(I:C) (HMW), poly(dA:dT) or VSV-eGFP. (D) Real-time PCR analysis of IFNB, IFIT2 and CCL5 mRNA in 293T cells treated with Usp3-specific or control shRNA, followed by the same treatment set as in C. (E) Enzyme-linked immunosorbent assay of IFN- $\beta$ protein in 293T cells, THP-1 and PBMCs treated with Usp3-specific or control siRNA or shRNA, followed by the VSV-eGFP infection. (F, G) Phase-contrast $(\mathrm{PH})$ and fluorescence microscopy $(\mathbf{F})$ and flow cytometry $(\mathbf{G})$ assessing the infection of 293T cells left untreated or treated with Usp3-specific or control siRNA, and then infected with VSVeGFP at an $\mathrm{MOI}$ of 0.05 . Original magnification $(\mathbf{F}), \times 10$. Numbers above bracketed lines $(\mathbf{G})$ indicate the percentage of cells expressing eGFP (infected cells). Data in panels $\mathbf{C}$-E are presented as the means \pm SD of three independent experiments. ${ }^{*} P<$ $0.05,{ }^{* *} P<0.01$ and ${ }^{* * *} P<0.001$, versus cells transfected with control siRNA (Student's $t$-test). 
together with increasing amounts of USP3 plus the ISRE luciferase reporter. We found that USP3 failed to inhibit ISRE-luc activity induced by MAVS, STING, TBK1, IRF3 or TIRF (Figure 3C and Supplementary information, Figure S3A-S3B). Similar results were obtained with IFN- $\beta$-luc reporter (Figure 3D). Consistent with these results, we found that knockdown of USP3 enhanced ISRE-luc or IFN- $\beta$-luc activity induced by RIGI $(\mathrm{N})$ and MDA5 $(\mathrm{N})$ but not by MAVS, TBK1 or IRF3 (Figure 3E and Supplementary information, Figure S3C).
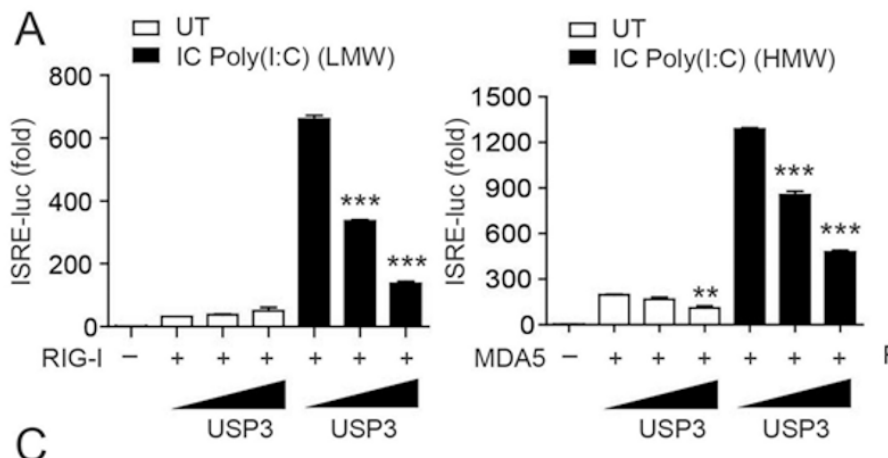

B

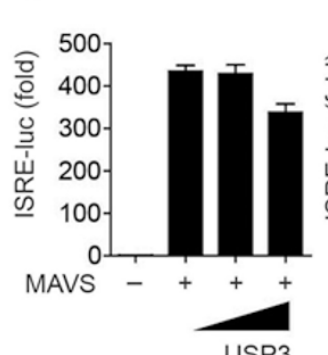

USP3

E

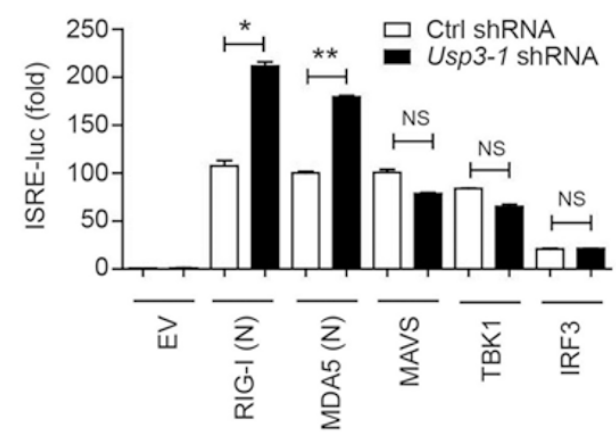

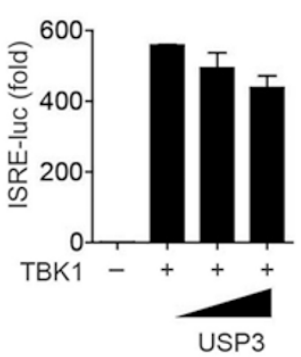

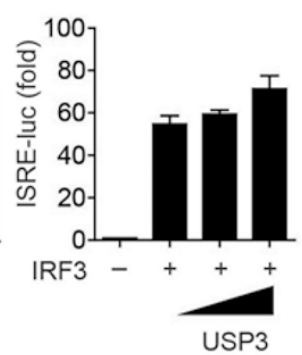

$\mathrm{F}$

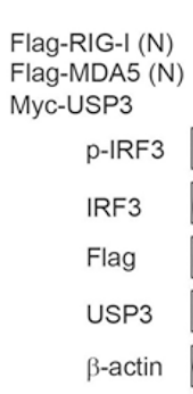

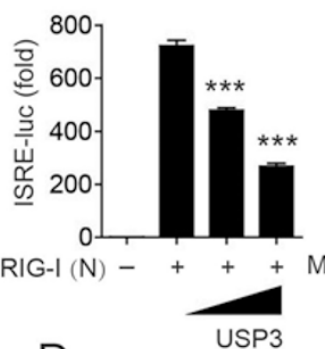

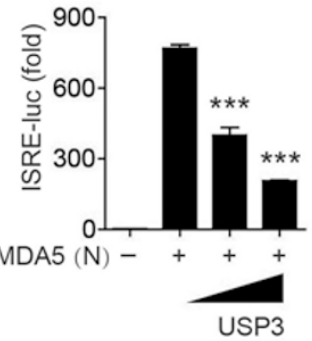

$\mathrm{D}$
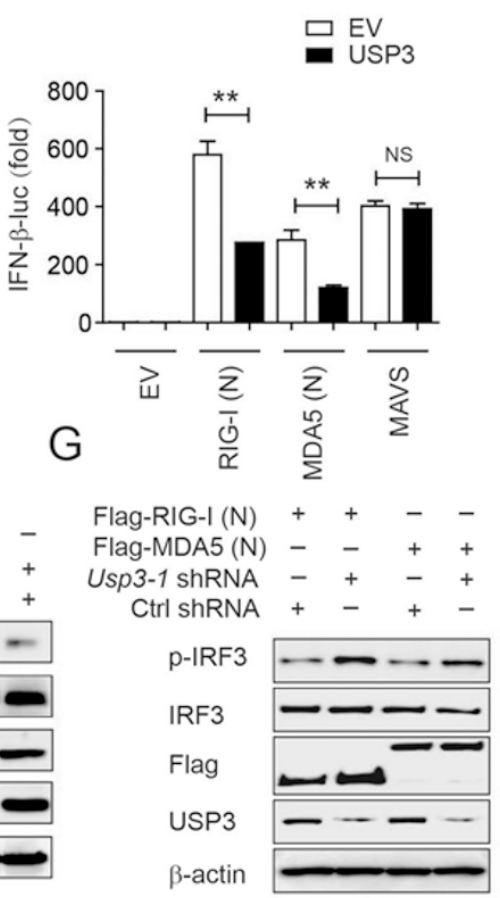

Figure 3 USP3 regulates type I IFN signaling through RIG-I and MDA5. (A) Luciferase activity in 293T cells transfected with plasmid encoding a luciferase reporter for ISRE together with empty vector (no wedge) or an expression vector for USP3 (0, 200 and $400 \mathrm{ng}$; wedge) and RIG-I or MDA5, followed by treatment with intracellular low molecular weight (LMW) poly(I:C) (1 $\mu \mathrm{g} / \mathrm{ml})$ or high molecular weight $(\mathrm{HMW})$ poly $(\mathrm{l}: \mathrm{C})(1 \mu \mathrm{g} / \mathrm{ml})$. Results are presented relative to Renilla luciferase activity. (B, C) USP3 inhibits RIG-I-, MDA5-mediated ISRE activation but not MAVS-, TBK1- and IRF3-mediated ISRE activation. 293T cells were transfected with an ISRE luciferase reporter, together with vector for RIG-I (N), MDA5 (N) (B), MAVS, TBK1 or IRF3 (C), along with empty vector (no wedge) or increasing amounts (wedge) of expression vector for USP3. (D) USP3 inhibits RIG-Iand MDA5-mediated activation but not MAVS-mediated activation of the IFN- $\beta$ promoter. The experiments were similarly performed as in B. (E) Knockdown of USP3 enhances RIG-I- and MDA5-mediated ISRE activation but not MAVS-, TBK1- and IRF3-mediated ISRE activation. 293T cells were transfected with an ISRE luciferase reporter, together with vector for RIG-I (N), MDA5 (N), MAVS, TBK1 or IRF3, along with control (ctrl) shRNA or Usp3-specific shRNA. (F, G) Immunoblot analysis of total and phosphorylated (p-) IRF3 in 293T cells transfected with various combinations (above lanes) of plasmid for Flag-tagged RIG-I (N) or MDA5 (N) plus vector for Myc-tagged USP3 (F) or USP3 shRNA and control shRNA (G). Data in panels A-E are presented as the means \pm SD of three independent experiments. ${ }^{*} P<0.05$, ${ }^{* *} P<0.01$ and ${ }^{* * *} P<0.001$, versus overexpression of RIG-I, MDA5, MAVS, TBK1 or IRF3 alone (Student's $t$-test). 
Furthermore, overexpression of USP3 inhibited IRF3 phosphorylation induced by RIG-I (N) or MDA5 (N), but not by MAVS or IRF3, while knockdown of USP3 had the opposite effects (Figure $3 \mathrm{~F}$ and $3 \mathrm{G}$ and Supplementary information, Figure S3D). Furthermore, unlike CYLD that inhibited type I IFN signaling activated by RIG-I and MAVS, USP3 inhibited type I IFN signaling induced by RIG-I but not by MAVS (Supplementary information, Figure S3E). These results suggest that USP3 directly inhibits IRF3 phosphorylation and type I IFN signaling through RLRs.

\section{USP3 interacts with RIG-I and MDA5 after ligand stimu- lation}

As USP3 has been reported to be in the nucleus to modify the chromatin [23], we examined whether USP3 can translocate to the cytoplasm when type I IFN signaling is activated. In cells co-transfected with GFP-USP3 and dsRed-RIG-I, USP3 was exclusively localized in the nucleus, while RIG-I was in the cytoplasm. However, after treatment with intracellular poly(I:C), some USP3 protein was translocated from the nucleus to the cytoplasm and co-localized with RIG-I (Supplementary information, Figure S4A). We next sought to determine whether USP3 could directly interact with RIG-I or MDA5. To address this issue, we transfected 293T cells with Flag-tagged $R I G-I$ together with Myc-tagged USP3 and then treated the cells with intracellular poly(I:C) (LMW or HMW). Coimmunoprecipitation and immunoblot analyses revealed that USP3 did not interact with RIG-I in unstimulated cells, but interacted with RIG-I after ligand stimulation, and the interaction peaked at 6 and $8 \mathrm{~h}$ after poly(I:C) (LMW) treatment (a ligand for RIGI) (Figure 4A). Similarly, USP3 interacted with MDA5 but not RIG-I after poly(I:C) (HMW) treatment (a ligand for MDA5) (Figure 4A and Supplementary information, Figure S4B). By contrast, USP3 did not interact with any downstream signaling proteins, including MAVS, TBK1, IKKi, IRF3, TRAF3 or TRAF6 after ligand stimulation (Supplementary information, Figure S4C-S4D). These results suggest that USP3 specifically interacts with active form of RIG-I or MDA5.

To determine the physiological relevance of these findings, we treated the THP-1 cells with intracellular poly(I:C) (LMW, HMW), poly(dA:dT) or infected the THP-1 cells with VSV-eGFP, and then collected cells at various time points. Immunoprecipitation and western blot analyses revealed that USP3 did not interact with either RIG-I or MDA5 in unstimulated THP-1 cells. However, USP3 strongly interacted with RIG-I, but only weakly interacted with MDA5, after Poly(I:C) (LMW) treatment or VSV-eGFP infection (Figure 4B). Converse- ly, poly(I:C) (HMW) treatment induced the strong interaction of USP3 with MDA5, but little with RIG-I (Figure 4B). We only observed a weak interaction between USP3 and RIG-I, but no interaction between USP3 and MDA5 after poly(dA:dT) stimulation (Supplementary information, Figure S4E), simply because poly(dA:dT) treatment mainly activates DDX41- and IFI16-mediated pathway in THP-1 cells $[9,10]$. To further assess whether USP3 interacts with RLRs in primary cells, we freshly isolated PBMCs and then infected them with VSV-eGFP. We found that USP3 interacted with RIG-I, but not with MDA5 in PBMCs $8 \mathrm{~h}$ post VSV-eGFP infection (Figure $4 \mathrm{C})$. Taken together, these results indicate that USP3 interacts with RIG-I or MDA5 in a ligand-dependent manner.

To identify the domain of RLRs responsible for interaction with USP3, we generated full-length RIG-I, RIGI $(\mathrm{N})$ and RIG-I without CARD (RIG-I- $\Delta \mathrm{N})$ and tested their ability to interact with USP3 (Figure 4D). We found that USP3 had weak interaction with full-length RIG-I, strong interaction with RIG-I $(\mathrm{N})$, but no interaction with RIG-I $(\Delta N)$ (Figure 4D). Similar results were obtained with MDA5, showing that USP3 weakly interacted with full-length MDA5 and strongly interacted with MDA5 $(\mathrm{N})$, but not with other MDA5 mutants (Figure 4E). Thus, specific interaction of USP3 with the CARD of RIG-I (i.e., RIG-I (N)) or MDA5 (i.e., MDA5 (N)) is consistent with functional inhibition results shown in Figure 3A and 3B, indicating that USP3 specifically binds to the CARD of RLRs only when they are exposed.

\section{USP3 deubiquitinates K63-linked polyubiquitin chains on $R I G-I$}

As it is known that K63-linked ubiquitination of RIGI is essential for its activation to trigger the production of type I IFNs [2], we next asked whether USP3 has deubiquitinating enzymatic activity on RIG-I ubiquitination. Coimmunoprecipitation and immunoblot analysis showed that overexpression of USP3 potently inhibited total ubiquitination and K63-linked ubiquitination of RIG-I (N), but has little or no effects on the K48-linked ubiquitination of RIG-I (N) (Figure 5A and Supplementary information, Figure S5A-S5B). To further define the role of USP3, we transfected 293 T cells with HA-K63Ub, Flag-RIG-I, Myc-USP3 or empty vector, and then treated the cells with intracellular poly(I:C) (LMW). As shown in Figure 5B, RIG-I was highly ubiquitinated with K63-linkage from 4 to $8 \mathrm{~h}$ post stimulation. However, ectopic expression of USP3 markedly inhibited the K63linked ubiquitination on RIG-I (Figure 5B). Consistent with that observation, knockdown of USP3 increased K63-linked ubiquitination on RIG-I after stimulation 
A

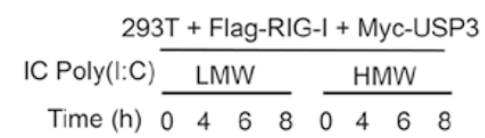

Time (h) $\begin{array}{lllllllll}0 & 4 & 6 & 8 & 0 & 4 & 6 & 8\end{array}$

IP: $\alpha$-Flag

IB: $\alpha-$ Myc

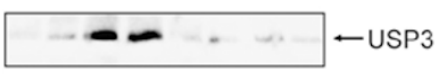

WCL IB: $\alpha$-Myc

PBMC + VSV-eGFP

C

Time (h) $\frac{0 \quad 824}{0.07}$

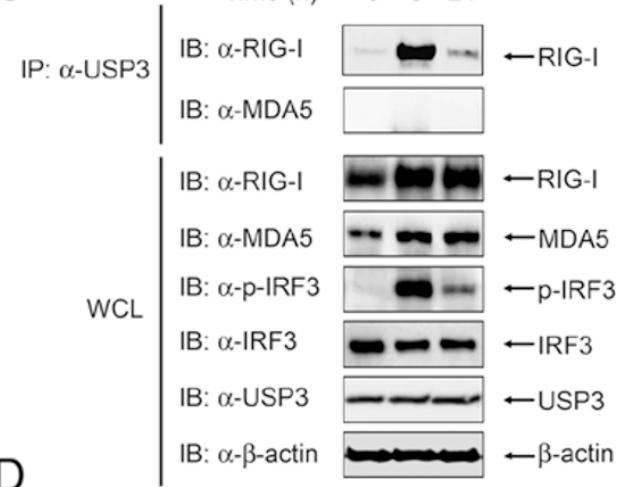

IP: $\alpha$-USP3 $\mid \begin{array}{ll}\text { IB: } \alpha \text {-RIG-I } \\ \text { IB: } \alpha \text {-MDA5 }\end{array}$

B

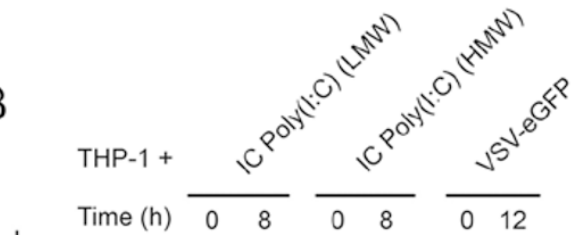

$\mathrm{D}$
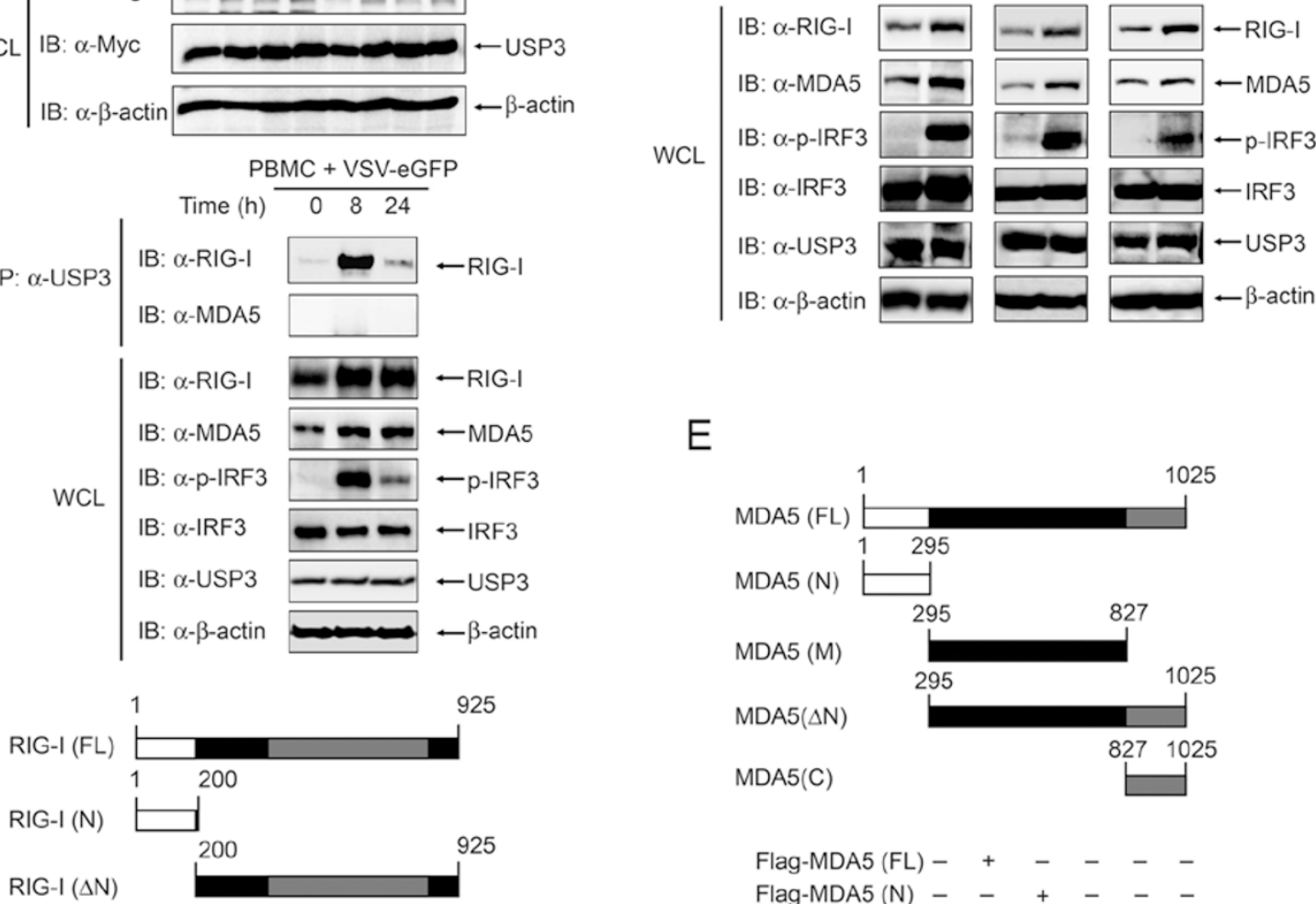

E
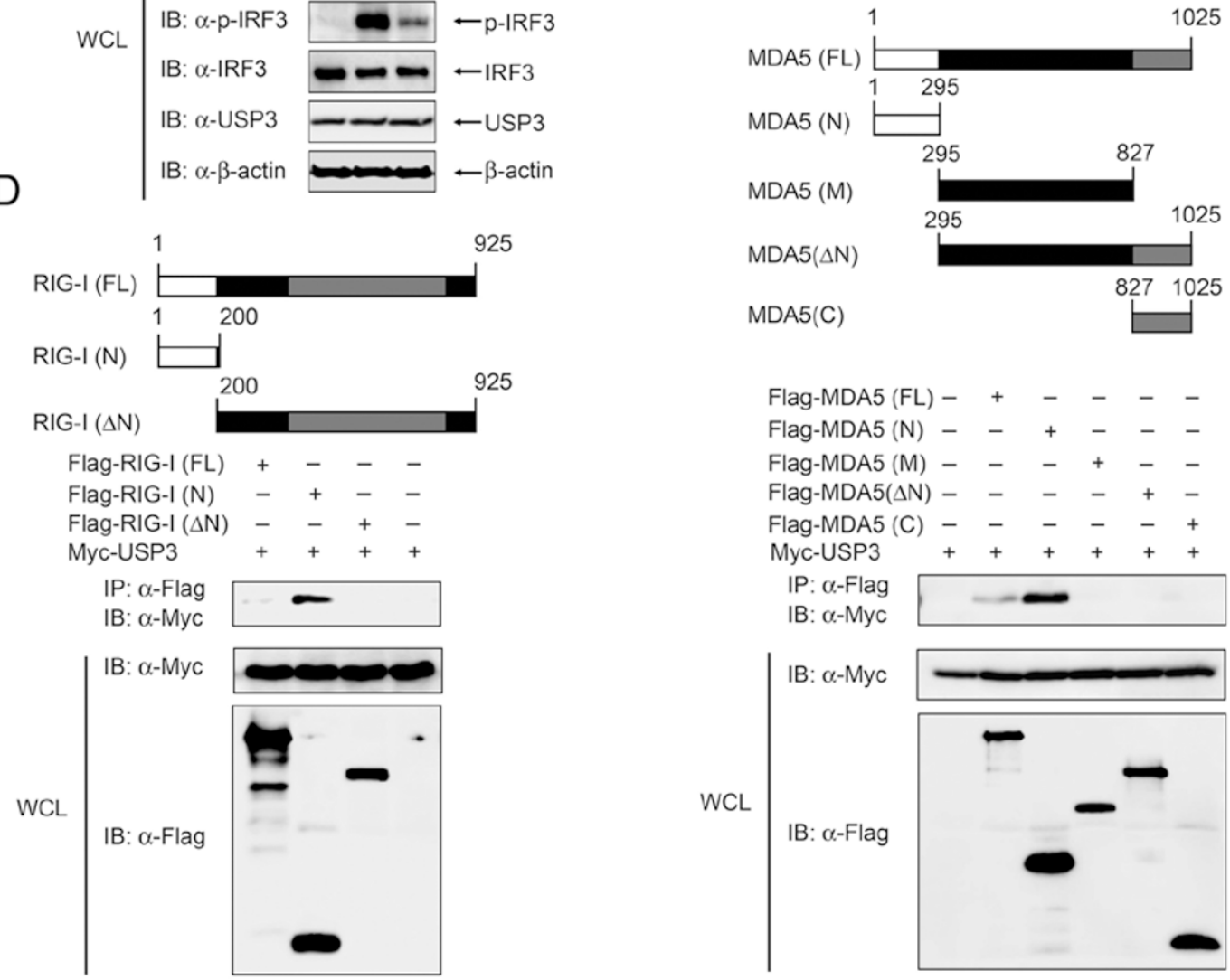

Figure 4 USP3 interacts with RIG-I and MDA5 after dsRNA stimulation and viral infection. (A) 293T cells were transfected with vectors for Myc-USP3 and Flag-RIG-I, followed by the treatment with intracellular Poly(I:C) and immunoprecipitation (IP) with anti-Flag beads and immunoblot analysis with anti-Myc. WCL, immunoblot analysis of whole-cell lysates without immunoprecipitation (throughout). (B, C) Extracts of THP-1 cells (B) or PBMCs (C) infected with VSV-eGFP for various times (above lanes) or treated with intracellular Poly(I:C) were subjected to immunoprecipitation with anti-USP3 and immunoblot analysis (antibodies, shown on the left). (D, E) Coimmunoprecipitation and immunoblot analysis of 293T cells transfected with deletion mutants of RIG-I (D) or MDA5 (E) along with vector for Myc-USP3. 
A

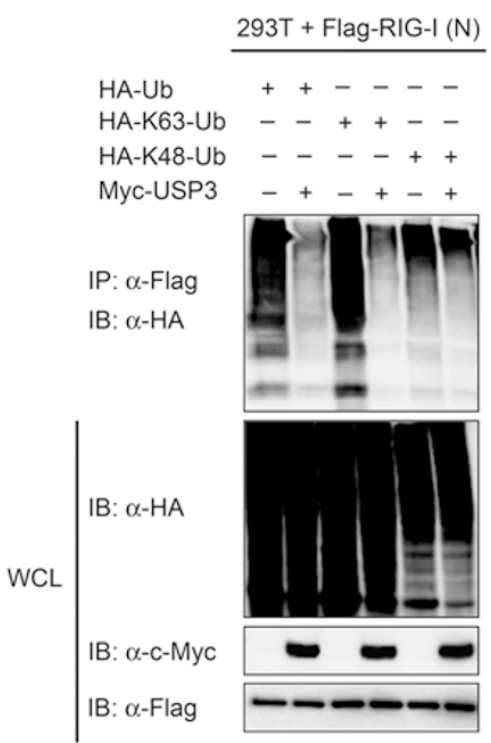

C

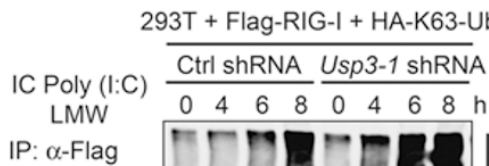

IB: $\alpha-\mathrm{HA}$

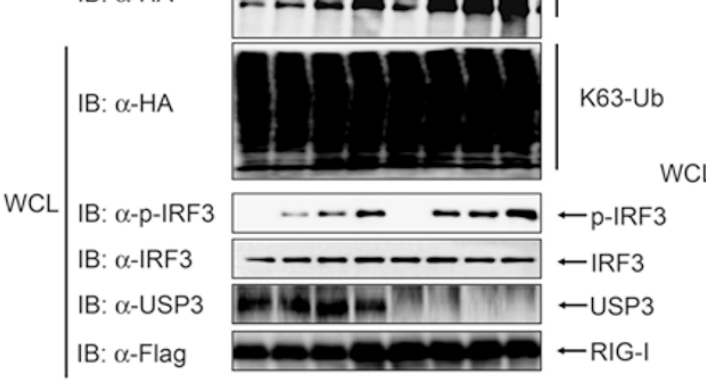

$\mathrm{D}$
B
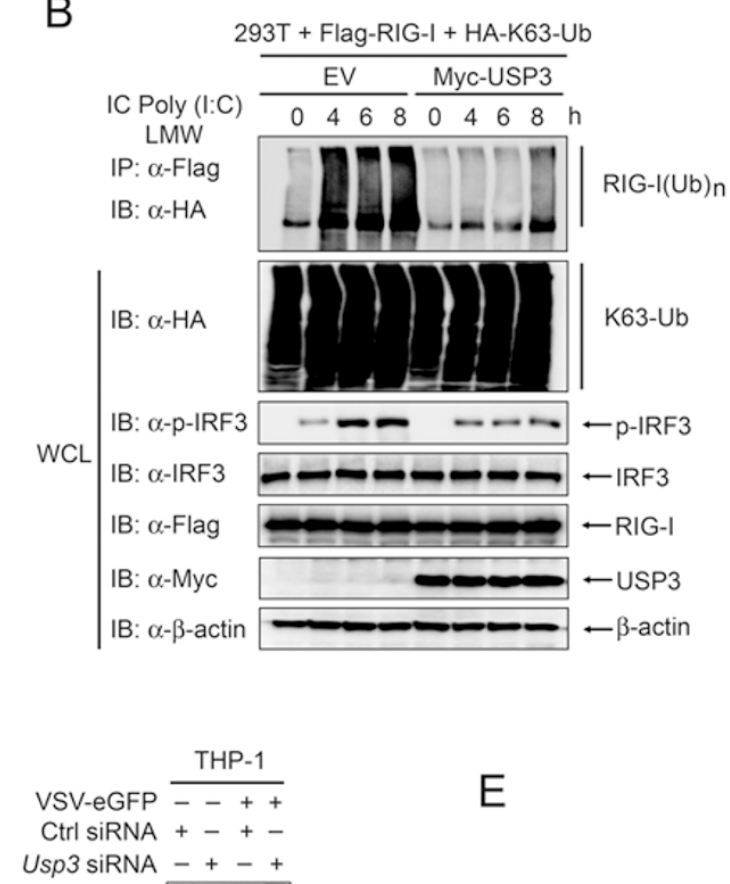

IP: $\alpha$-RIG-I IB: $\alpha-K 63-U b$

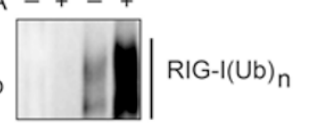

$\mathrm{E}$

IB: $\alpha$-Ub
IB: $\alpha$-p-IRF3
IB: $\alpha$-IRF3
IP: $\alpha$-RIG-I
IB: $\alpha$-USP3
IB: $\alpha-\beta$-actin

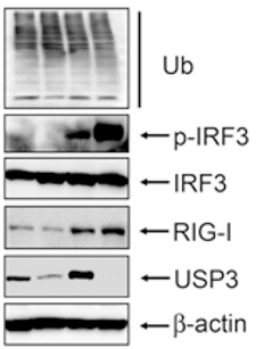

Flag-RIG-I + +

$\mathrm{HA}-\mathrm{K} 63-\mathrm{Ub}++$

Flag-USP3 - +

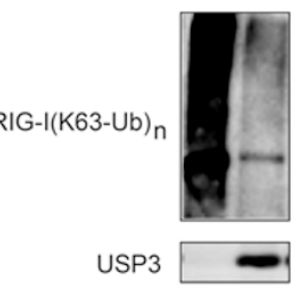

Figure 5 USP3 inhibits IRF3 activation by removing K63-linked ubiquitin chain on RIG-I. (A) Lysates of 293T cells transfected with plasmid for Flag-RIG-I (N) and HA-tagged ubiquitin (HA-Ub), HA-tagged K63-linked ubiquitin (HA-K63-Ub) or HA-tagged K48-linked ubiquitin (HA-K48-Ub), together with the empty vector or expression vector of Myc-USP3 were immunoprecipitated with anti-Flag and immunoblotted with anti-HA. (B) Lysates of 293T cells transfected with plasmid for HA-K63-Ub and FlagRIG-I, together with the empty vector or expression vector of Myc-USP3, and treated with intracellular poly(I:C) LMW were subjected to immunoprecipitation with anti-Flag and immunoblot analysis with anti-HA. (C) Lysates of 293T cells transfected with plasmid for HA-K63-Ub, Flag-RIG-I, together with the control shRNA or USP3-specific shRNA, and treated with intracellular poly(I:C) LMW were immunoprecipitated with anti-Flag and immunoblotted with anti-HA. (D) Extracts of THP-1 cells transfected with control or USP3-specific siRNA and infected with VSV-eGFP were subjected to immunoprecipitation with anti-RIG-I and immunoblot analysis with antibody against K63-linked ubiquitin. (E) Ubiquitinated RIG-I was incubated with immunopurified Flag-USP3 in vitro in deubiquitinating buffer. The immunoblot was probed with anti-HA.

(Figure 5C). Similar inhibitory effects of USP3 expression on MDA5 ubiquitination were observed when MDA5 and its ligand were tested (Supplementary information, Figure S5C-S5D). We did not observe any effect of USP3 expression on ubiquitination of MAVS, TBK1, TRAF3 or IRF3 (Supplementary information, Figure $\mathrm{S} 5 \mathrm{E})$. These results suggest that USP3 specifically inhib- its K63-linked polyubiquitination of RLRs after ligand stimulation.

To substantiate these findings, we knocked down endogenous USP 3 in THP-1 cells and assessed RIGI ubiquitination after viral infection. In cells transfected with USP3 siRNA, we observed increased K63-linked polyubiquitination of RIG-I (but not K48-linked RIG- 
I polyubiquitination), compared with cells transfected with control siRNA after viral infection (Figure 5D and Supplementary information, Figure S5F). Interestingly, increased K63-linked ubiquitination of RIG-I was correlated with enhanced phosphorylation of IRF3 (Figure 5D). In addition, knockdown of USP 3 also enhanced ubiquitination of MDA5 after intracellular poly(I:C) treatment in THP-1 cells (Supplementary information, Figure S5G). To test whether USP3 can directly deubiquitinate K63-ubiqutinated RIG-I or MDA5, we determined the activity of purified USP3 protein on ubiquitinated RIG-I or MDA5 in vitro. As shown in Figure 5E and Supplementary information, Figure S5H, the co-incubation of ubiquitinated RIG-I or MDA5 with the purified USP3 protein reduced the levels of polyubiquitin chains on RIG-I or MDA5. Taken together, these data suggest that USP3 directly cleaves K63-linked polyubiquitin chains on RLRs and thus inhibits IFN- $\beta$ signaling.

\section{Both zinc-finger Ub-binding and USP catalytic domains of USP 3 are required for its deubiquitinating activity}

USP3 contains two conserved protein domains: a zincfinger Ub-binding domain (ZnF-UBP) and a catalytic domain of the Ub-specific protease (USP) class [23]. To identify which domain of USP3 is responsible for RLR deubiquitination, we generated two deletion mutants containing the ZnF-UBP domain (ZnF) or USP catalytic domain (UCH) of USP3, and assessed their ability to inhibit RIG-I-induced signaling pathway (Figure 6A). Like full-length USP3, USP3 (UCH) interacted with RIG-I (N) and MDA5 $(\mathrm{N})$, but USP3 $(\mathrm{ZnF})$ failed to do so (Figure 6B). However, USP3 (UCH) showed only partial inhibitory effect on ISRE-luc activation, compared with full-length USP3, which strongly inhibited RIG-I (N)-induced activity of ISRE-luc reporter (Figure 6C). USP3 (ZnF) did not have any inhibitory effect on RIG-I (N)-induced ISRE-luc activation (Figure 6C). Consistent with these results, we found that USP3 (ZnF) could not block RIG-I (N) ubiquitination, while USP3 (UCH) only partially blocked RIG-I (N) ubiquitination, compared to full-length USP3 (Supplementary information, Figure $\mathrm{S6A}$ ). These results suggest that although $\mathrm{ZnF}$ domain of USP3 itself cannot inhibit RIG-I-induced type I IFN activation, it may be required for the $\mathrm{UCH}$ domain to achieve the maximal catalytic activity.

To further investigate whether both $\mathrm{ZnF}$ domain and $\mathrm{UCH}$ domain of USP3 are required for the inhibitory function of USP3, we substituted a serine residue for cysteine within USP3 catalytic domain (C168S), thus generating an inactive USP3 mutant, while substitution of the conserved histidine residue with alanine at position 56 within the $\mathrm{ZnF}$ domain (H56A) gave rise to a USP3 mutant with disrupted $\mathrm{ZnF}$ structure. In vitro ubiquitination assay showed that neither USP3 (C168S) mutant nor USP3 (H56A) mutant removed the K63-linked polyubiquitin chain from RIG-I (Figure 6D), indicating that both intact $\mathrm{ZnF}$ and catalytic domains are required for RIGI deubiquitination. To further determine whether USP3 (C168S) and USP3 (H56A) mutants affect the inhibitory function of USP3 on type I IFN signaling, we performed ISRE-luc assays and found that both mutations abrogated the ability of USP3 to inhibit ISRE-luc activity induced by intracellular poly(I:C) (LMW or HMW) treatment as well as by the ectopic expression of RIG-I (N) or MDA5 (N) (Figure 6E and 6F). Combined mutations of these two amino acids had the same effect on ISRE-luc activity as their single-mutation constructs (Supplementary information, Figure S6B). Interestingly, overexpression of these USP3 mutants could block the inhibitory function of wild-type (WT) USP3 (Supplementary information, Figure S6C). In addition, USP3 (C168S) mutant inhibited USP3-mediated deubiquitination of RIG-I (Supplementary information, Figure S6D). Taken together, these results suggest that both cysteine 168 and histidine 56 of USP3 are essential for its deubiquitinating activity.

\section{USP3 specifically binds to the ubiquitinated RIG-I and} cleaves polyubiquitin chains

To understand the molecular mechanisms by which USP3 binds to the activated form of RIG-I and inhibits type I IFN signaling, we reasoned that USP3 might bind to RIG-I through K63-linked polyubiquitin chains. To test this hypothesis, we performed immunoprecipitation and immunoblot analyses and found that the K63-linked ubiquitination of RIG-I (N) was much stronger in the cells expressing USP3 (C168S) or USP3 (H56A) mutant than in cells expressing WT USP3 (Figure 7A and Supplementary information, Figure S7A). More importantly, immunoprecipitation of Flag-tagged RIG-I (N) with antiFlag and immunoblot analysis of Myc-tagged USP3 WT and mutants with anti-Myc revealed that interaction of RIG-I (N) with USP3 (C168S) or USP3 (H56A) was much stronger than with WT USP3 (Figure 7A). The correlation between RIG-I (N) ubiquitination and interaction of USP3 as well as its mutants with RIG-I (N) suggest that USP3 may only interact with the ubiquitinated RIGI. To test this hypothesis, we generate K172R mutant of RIG-I, which could abrogate the majority of K63-linked ubiquitination of RIG-I [21]. We co-transfected the 293T cells with vectors for HA-K63-Ub, Flag-RIG-I or FlagRIG-I (K172R), together with or without Myc-USP3, and then treated the cells with intracellular poly(I:C) (LMW). As expected, WT RIG-I underwent heavy K63-linked ubiquitination after poly(I:C) stimulation, and USP3 in- 

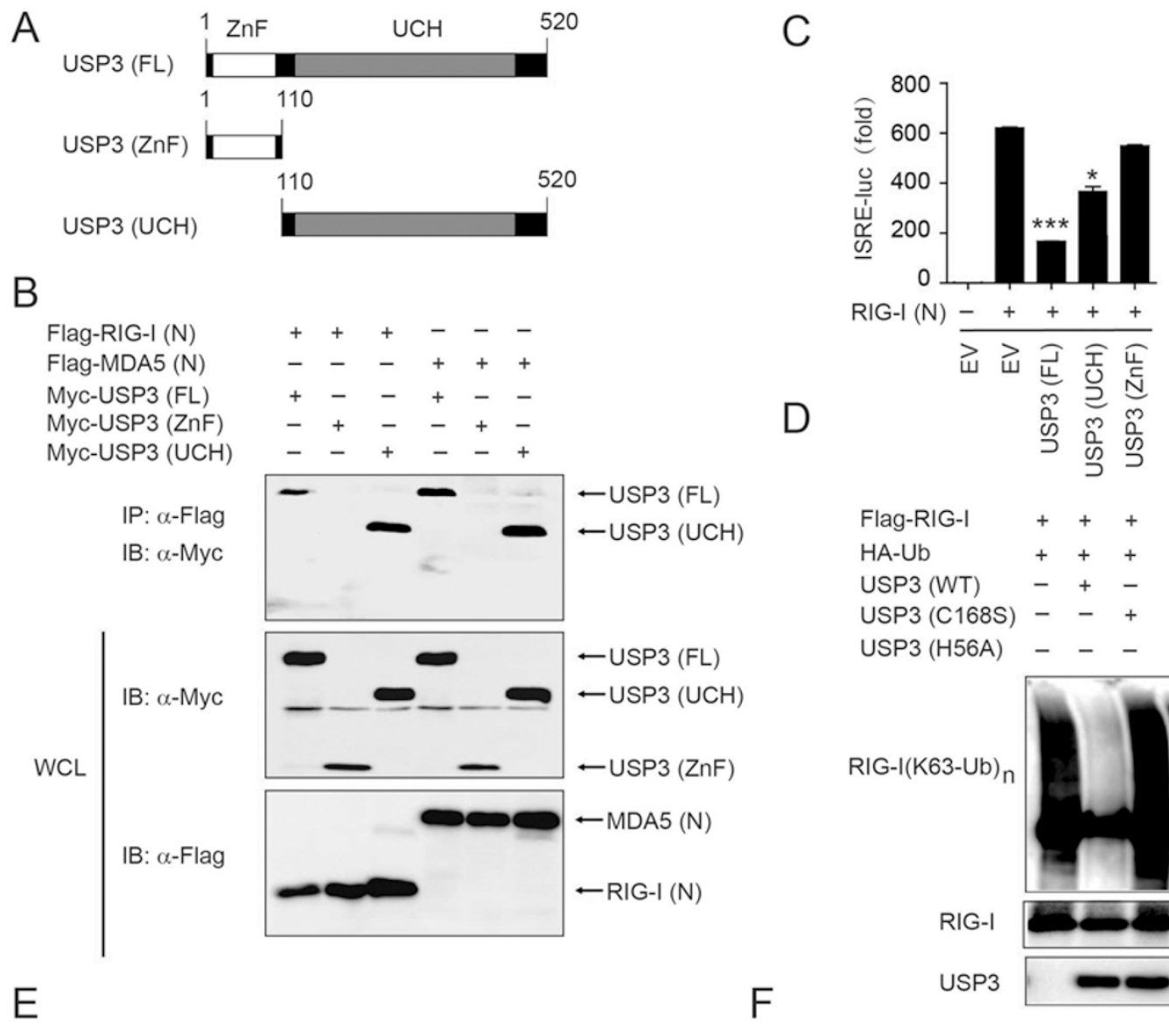

E

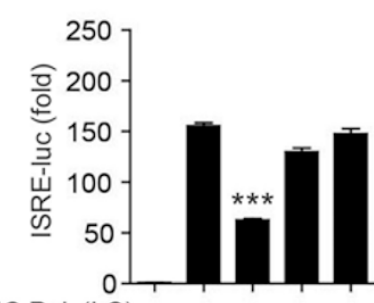

IC Poly (I:C) LMW

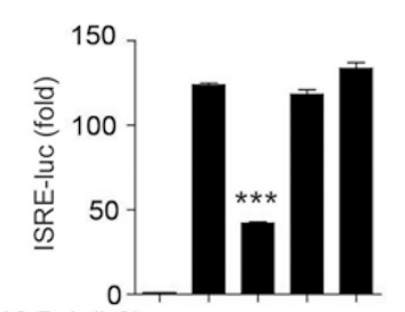

IC Poly(I:C) HMW
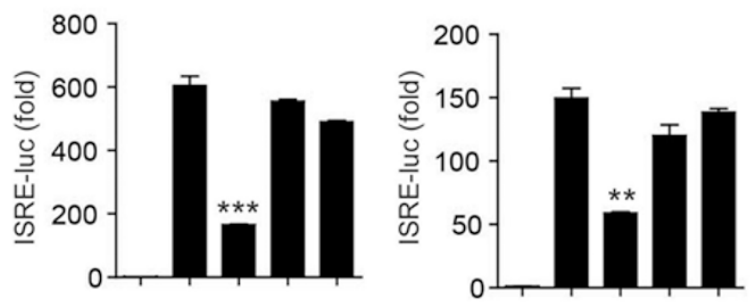

RIG-I (N)

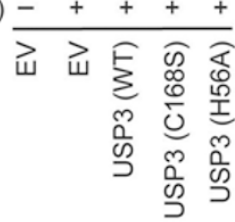

$\operatorname{MDA} 5(\mathrm{~N})$

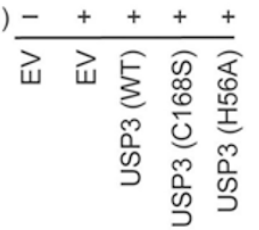

Figure 6 Both $\mathrm{ZnF}$ and $\mathrm{UCH}$ domains are required for USP3-mediated inhibition of type I interferon signaling. (A) Constructs of full-length USP3 (USP3 (FL)) or USP3 containing only ZnF (USP3 (ZnF)) or UCH domain (USP3 (UCH)). (B) Coimmunoprecipitation and immunoblot analysis of 293T cells transfected with various combinations (above lanes) of plasmid for Flag-RIG-I (N), Flag-MDA5 (N) and the Myc-tagged USP3 constructs shown in A. (C) Luciferase activity of 293T cells transfected with expression vector for RIG-I $(N)$ and an ISRE luciferase reporter, together with empty vector (EV) or vectors for the USP3 constructs. (D) Ubiquitinated RIG-I was incubated with immunopurified Flag-USP3, Flag-USP3(C168S) or Flag-USP3 (H56A) in vitro in deubiquitinating buffer. The immunoblot was probed with anti-K63-ubiquitin. (E, F) Luciferase activity of 293T cells treated with intracellular poly(I:C) (E) or transfected with expression vector for RIG-I (N) or MDA5 (N) (F) and an ISRE luciferase reporter, together with empty vector (EV) or vectors for the USP3 mutants. Data in panels $\mathbf{C}$, $\mathbf{E}$ and $\mathbf{F}$ are reported as the means $\pm \mathrm{SD}$ of three independent experiments. ${ }^{*} P<0.05,{ }^{* *} P<0.01$, ${ }^{* *} P<0.001$, versus control cells expressing $R I G-I(N)$ or treated with intracellular poly(I:C) alone. 


\section{A}

\begin{tabular}{|c|c|c|c|}
\hline Flag-RIG-I (N) & & + & \\
\hline HA-K63-Ub & & + & \\
\hline Myc-USP3 (WT) & & + & \\
\hline Myc-USP3 (C168S) & & - & \\
\hline Myc-USP3 (H56A) & & - & \\
\hline
\end{tabular}

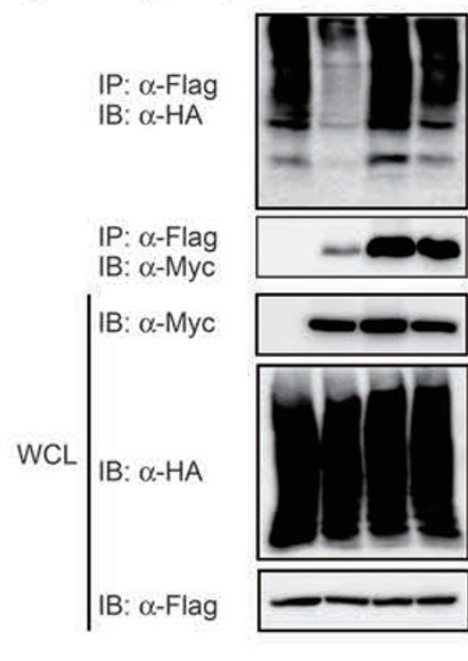

C

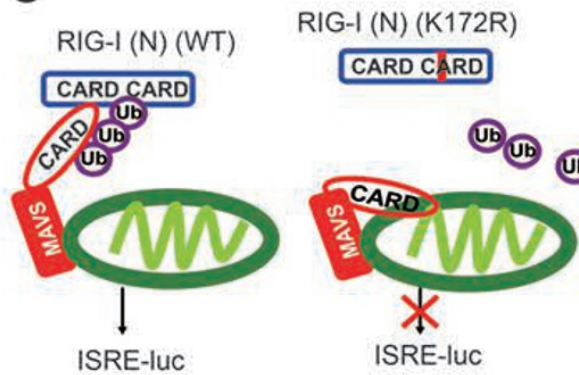

E

HA-K63-Ub + + + Myc-USP3 (C168S) + + +

Flag-RIG-I(N) WT K172R D122A

IP: $\alpha$-Flag

IB: $\alpha$-Myc

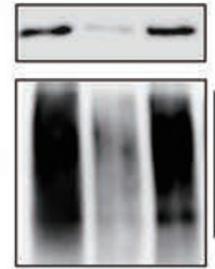

WCL $\mid \begin{aligned} & \text { IB: } \alpha-\text {-Myc } \alpha-\mathrm{HA} \\ & \text { IB: } \alpha-\text { Flag }\end{aligned}$

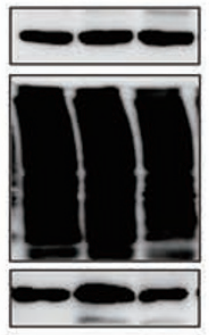

B
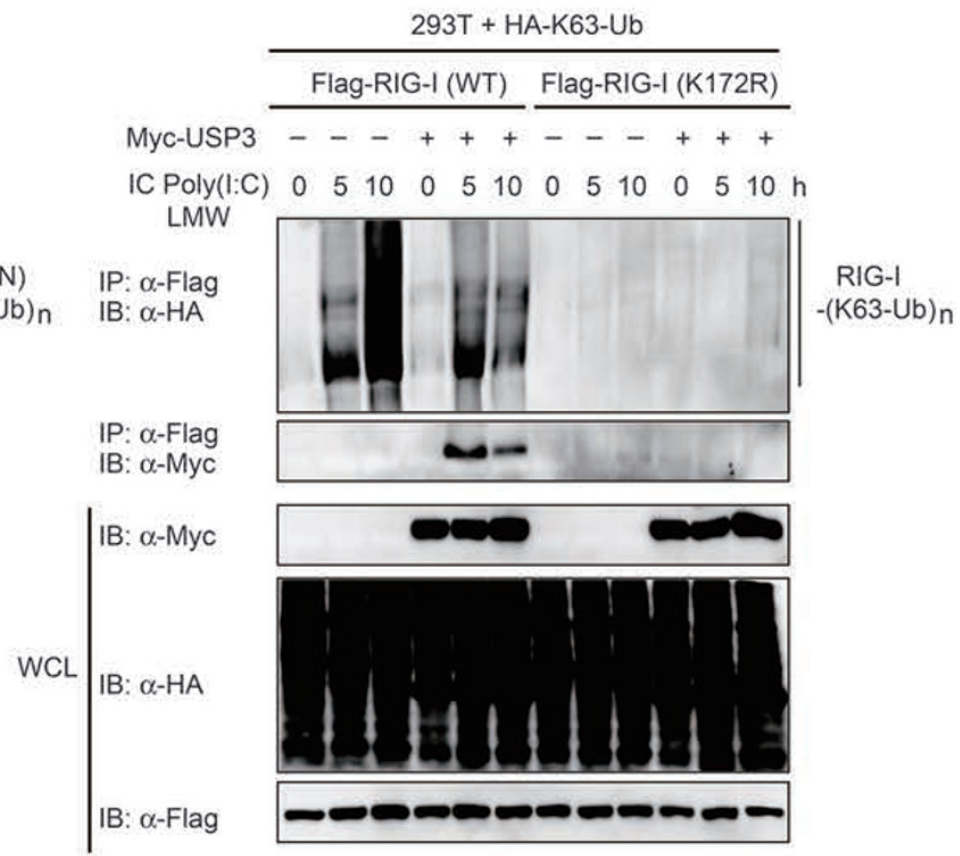

D

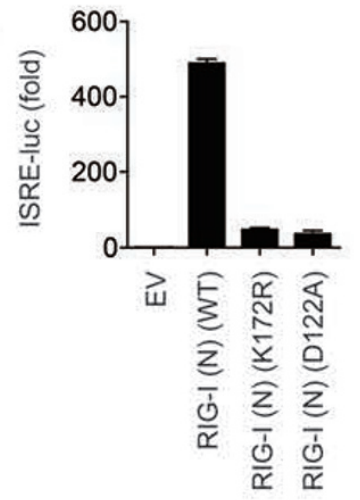

F

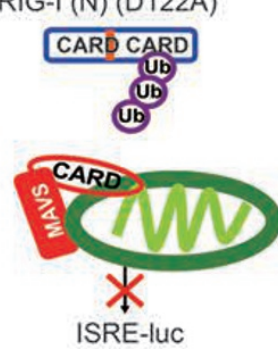

c-Myc-USP3

RIG-I (N)

$-(\mathrm{K} 63-\mathrm{Ub}) \mathrm{n}$

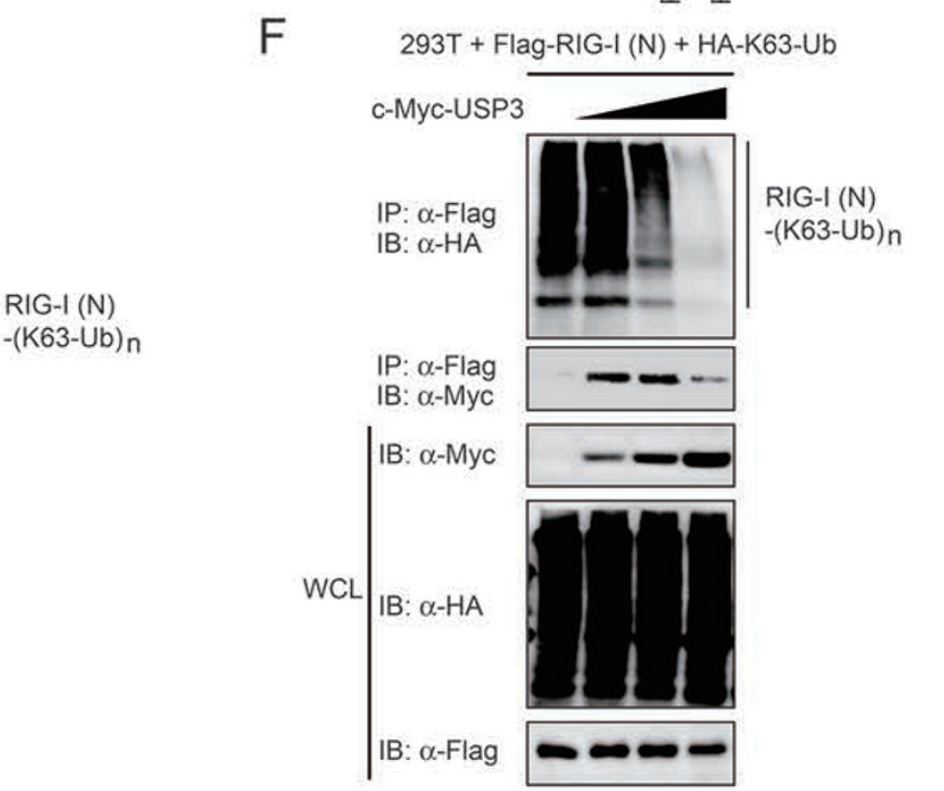


teracted with WT RIG-I to inhibit its polyubiquitination (Figure 7B). By contrast, RIG-I (K172R) mutant could not be ubiquitinated after stimulation. There was no detectable interaction between USP3 and RIG-I (K172R) (Figure 7B). As there are many signaling proteins, such as TRAF3 and TRAF6 that undergo robust K63-linked ubiquitination after TLR stimulation, we further investigated whether USP3 interacts with the K63-linked polyubiquitin chains of other proteins. We found that although TRAF3 and TRAF6 are heavily ubiquitinated with a K63 linkage, USP3 could not bind to K63-ubiquitinated TRAF3 or TRAF6 (Supplementary information, Figure S7B), suggesting that USP3 only interacts with the K63ubiquitinated RIG-I.

To determine whether the interaction between USP3 and RIG-I was solely dependent on the ubiquitination status of RIG-I, we generated two RIG-I (N) mutants and predicted on the basis of previous study [34] that substitution of Lys172 with arginine residue (K172R) of RIGI $(\mathrm{N})$ could abrogate the K63-linked ubiquitination of RIG-I protein, while substitution of Asp122 with alanine (D122A) of RIG-I (N) does not affect the ubiquitination of RIG-I, but inhibits type I IFN activation by blocking the interaction between RIG-I and MAVS (Figure 7C). Indeed, we found that neither K172R nor D122A mutants of RIG-I (N) could activate downstream type I IFN signaling (Figure 7D). USP3 could not interact with RIGI (K172R), which was weakly ubiquitinated. By contrast, USP3 could still strongly interact with RIG-I (D122A) mutants, which was heavily ubiquitinated, although it failed to activate MAVS and type I IFN signaling (Figure 7E). Taken together, these results suggested that USP3 interacts with ubiquitinated RIG-I, regardless of its ability to activate type I IFN signaling.

During our experiments, we consistently observed that the interaction of RIG-I with USP3 inactive mutants (C168S or H56A) is much stronger than its interaction with WT USP3. One plausible explanation is that WT USP3 may remove the polyubiquitin chains on RIG-I in a 'cut and run' manner, while its inactive mutants cannot remove polyubiquitin chains and lock on the polyubiquitin chains of RIG-I. To test this hypothesis, we co-transfected $293 \mathrm{~T}$ cells with vectors for HA-K63Ub, Flag-RIG-I $(N)$ and increasing amounts of $M y c-$ USP3. Coimmunoprecipitation and immunoblot analyses showed that when the amounts of polyubiquitin chains on RIG-I $(\mathrm{N})$ were decreased with increasing amount of USP3, the interaction between USP3 and RIG-I (N) was also reduced (Figure 7F). Similar results were obtained with USP3 and MDA5 (Supplementary information, Figure S7C), suggesting that once USP3 removes the polyubiquitin chains, it cannot bind to RIG-I or MDA5 protein any more.

\section{Discussion}

Because of the importance of type I IFN signaling pathway, it must be tightly regulated to maintain the balance between immunity and tolerance; otherwise, aberrant immune responses may occur, leading to severe or even fatal bacterial sepsis, autoimmune and chronic inflammatory diseases $[13,24]$. For this reason, many negative regulators have been identified to dampen type I IFN signaling through targeting different signaling molecules in the type I IFN signaling pathway. For example, NLRC5 and NLRX1 directly interact with RIG-I/MDA5 or MAVS to inhibit type I IFN signaling pathway [25-28]. PSMA7 and PCBP2/AIP4 inhibit type I IFN signaling by targeting MAVS for ubiquitination and degradation $[29,30]$. Similarly, NLRP4 inhibits type I IFN signaling by targeting TBK 1 for ubiquitination and degradation via a ubiquitin E3 ligase DTX4 [31]. Recently, several structural studies show that the activation of RIG-I is a two-step process and is tightly regulated through conformational changes $[18,19,32]$. In the absence of RNA ligand, RIG-I is in an autorepressed state. Upon RNA binding, RIG-I undergoes conformational changes to expose the CARD. Once the exposed CARD undergoes

Figure 7 USP3 binds to the ubiquitinated RIG-I and cleaves the K63-linked ubiquitin chains in a "cut and run" manner. (A) Extracts of 293T cells transfected with various combinations of plasmid for Flag-RIG-I (N), Myc-USP3 or it mutants, and HAtagged $K 63$ ubiquitin (HA-K63 Ub) were subjected to immunoprecipitation with anti-Flag beads and immunoblot analysis with anti-HA or anti-Myc. (B) Lysates of 293T cells transfected with plasmid for HA-K63-Ub and Flag-RIG-I or Flag-RIG-I (K172R), together with the empty vector or expression vector of Myc-USP3, and treated with intracellular poly(I:C) LMW were immunoprecipitated with anti-Flag and immunoblotted with anti-HA or anti-Myc. (C) Proposed model illustrating how RIG-I CARD and its mutants regulate type I IFN signaling pathways. (D) Luciferase activity of 293T cells transfected with expression vectors for RIG-I (N), RIG-I (N) (K172R) or RIG-I (N) (D122A) and an ISRE luciferase reporter. (E) Extracts of 293T cells transfected with various combinations of plasmid for Myc-USP(C168S), Flag-RIG-I (N) or its mutants, and HA-K63-Ub were subjected to immunoprecipitation with anti-Flag beads and immunoblot analysis with anti-HA or anti-Myc. (F) Extracts of 293T cells transfected with expression plasmid for Flag-RIG-I (N), HA-K63-Ub and increasing amount of Myc-USP3 (wedge) were subjected to immunoprecipitation with anti-Flag beads and immunoblot analysis with anti-HA or anti-Myc. 
K63-linked ubiquitination or directly interact with unanchored ubiquitin chains, the CARD of activated RIGI interacts with MAVS and activates the downstream signaling pathway. Several E3 ubiquitin ligases, including TRIM25 and Riplet, have been demonstrated to play a critical role in activation of RIG-I by catalyzing K63-linked ubiquitination of RIG-I [21, 33]. However, specific proteins that remove K63 polyubiquitin chains of RIG-I are not known. Our results presented in this study demonstrate that USP3 negatively regulates type I IFN signaling and antiviral immunity by removal of the K63-linked polyubiquitin chains from RIG-I. Ectopic expression of USP3 attenuated type I IFN signaling as well as antiviral immunity activated by intracellular viral RNA and VSV-eGFP. Conversely, knockdown of USP 3 enhanced virus-induced phosphorylation of IRF3 and expression of IFN- $\beta$ and IFN-stimulating genes, as well as cellular antiviral responses in a variety of human cells tested. Although K63-linked ubiquitination of MDA5 is evident in the $293 \mathrm{~T}$ cell-based overexpression system, endogenous K63-linked ubiquitination of MDA5 is barely detectable. However, unanchored K63-linked polyubiquitin chains have been reported to activate MDA5-mediated type I IFN signaling [34]. Therefore, it is possible that USP3 may bind to MDA5 with polyubiquitin chains without covalent linkage (unanchored) and then degrade polyubiquitin chains.

Although several DUBs, including A20 and CYLD, have been found to be involved in RIG-I-mediated type I IFN signaling, they are not specific for RIG-I. For example, A20 negatively regulates type I IFN signaling through a mechanism independent of its DUB enzyme activity [35]. CYLD can also inhibit type I IFN activation and remove the polyubiquitin chains from RIG-I, TBK1 and IKKi [36]. Indeed, our study shows that CYLD could inhibit type I IFN signaling activated by RIG-I and MAVS, whereas USP3 only inhibited type I IFN signaling induced by RIG-I, but not by MAVS, suggesting that USP3 specifically inhibit type I IFN signaling through RIG-I deubiquitination. To determine the specificity of USP3, we have provided several lines of evidence that USP3 specifically targets to the ubiquitinated RIG-I with a K63 linkage: (1) USP3 specially interacts with the active form of RIG-I or MDA5, but not downstream components MAVS, TRAF3, TRAF6, TBK1, IKKi or IRF3; (2) USP3 directly interacts with the CARD of RIG-I or MDA5; (3) endogenous USP3 interacts with RIG-I or MDA5 in a signal-dependent manner (only after ligand stimulation); (4) the intensity of the USP3-RLR interaction is correlated with the K63-linked ubiquitination of RLRs; and (5) USP3 failed to interact with RIG-I mutants that lose K63-linked polyubiquitination.
Our results demonstrate that USP3 can directly cleave the K63-linked polyubiquitin chains but not K48-linked polyubiquitin chains from RIG-I upon ligand stimulation or viral infection. Both ZnF-UBP domain and $\mathrm{UCH}$ domain of USP3 are critically required for its enzyme activity. The UCH domain of USP3 itself can interact with RIG-I, but its enzyme activity is much lower than full-length USP3, indicating that ZnF-UBP domain is also important for USP3 function. Although ZnF-UBP domain of USP3 cannot directly interact with RIG-I, the $\mathrm{ZnF}$ structure of this domain is essential to maintain the deubiquitinase activity of USP3. H56A mutant of USP3, which destroyed the $\mathrm{ZnF}$ structure, lost all of its enzyme activity as the inactive mutant of USP3 (C168S). Based on these experimental data, we propose a working model to illustrate how USP3 could negatively regulate RIG-Imediated type I IFN signaling (Supplementary information, Figure S7D). After viral infection, RIG-I first recognizes viral RNA and exposes its CARD, and then undergoes K63-linked ubiquitination through E3 ubiquitin ligases such as TRIM25, or directly binds to unanchored ubiquitin chains to become active. The activated RIGI in turn interacts with MAVS to trigger downstream signaling. Once RIG-I is activated (ubiquitinated), USP3 specifically binds to RIG-I and cleaves the K63-linked polyubiquitin chains, thus converting active RIG-I into its inactive form. Although USP3 mutant can still bind to ubiquitinated RIG-I, it fails to cleave the polyubiquitin chains, resulting in a stable USP3-ubiquitinated-RIG-I complex, which have negative-dominant inhibitory effect on WT USP3. There are several mechanisms operating in the cell to dampen RLR-mediated type I IFN signaling. For example, RNF125 inhibits type I IFN signaling by targeting RIG-I and MDA5 for degradation through K48-linked ubiquitination [37]. More recently, Siglec-G, a member of lectin family, has been identified to recruit SHP2 and the E3 ubiquitin ligase c-Cbl to RIG-I, resulting in RIG-I degradation via K48-linked ubiquitination [38]. In contrast, USP17 (also known as DUB3) has been identified to remove the K48-linked ubiquitin chains on RIG-I and MDA5, and promote virus-triggered signaling [39]. Thus, multiple negative regulatory mechanisms are exploited to tightly control type I IFN signaling.

In conclusion, we identify a previously unrecognized role of USP3 in the negative regulation of type I IFN signaling and antiviral immunity. USP3 specifically binds to ubiquitinated RLRs through the CARD and then cleaves the polyubiquitin chains. Our findings provide insights into the molecular mechanisms by which USP3 negatively regulates type I IFN signaling and thus plays a critical role in maintaining the balance between innate immune response and tolerance. 


\section{Materials and Methods}

\section{Cell culture and reagents}

HEK293T and THP-1cells (American Type Culture Collection) were maintained in DMEM (Mediatech) or RPMI-1640 medium (Invitrogen) containing 10\% heat-inactivated FCS. Buffy coats of blood from healthy donors (from the Gulf Coast Regional Blood Center) were used for isolation of PBMCs by density-gradient centrifugation with Lymphoprep (Nycomed Pharm). The use of PBMCs was in accordance with institutional guidelines and approved protocols by The Methodist Hospital Research Institute. Poly(I:C) (HMW), poly(I:C) (LMW) and poly(dA:dT) were purchased from Invivogen.

\section{Antibodies}

Anti-USP3 (sc-79318), anti-IRF3 (sc-9082) and anti-ubiquitin (sc-8017) were purchased from Santa Cruz Biotechnology; horseradish peroxidase(HRP)-anti-Flag (M2) (A8592) and anti- $\beta$-actin (A1978) were purchased from Sigma; HRP-antihemagglutinin (clone 3F10), anti-c-Myc-HRP (11814150001) and unlabeled anti-c-Myc (11667203001) were purchased from Roche Applied Science. Antibody to IRF3 phosphorylated at Ser396 (4947), anti-K63-ubiquitin (5621), anti-K48-ubiquitin (4289), antiRIG-I (3743 and 4200) and anti-MDA5 (5321) were from Cell Signaling Technology.

\section{Transfection and reporter assays}

HEK293T $\left(2 \times 10^{5}\right)$ cells were plated in 24-well plates and transfected, through the use of Lipofectamine 2000 (Invitrogen), with plasmids encoding an NF- $\mathrm{kB}$, IFN- $\beta$ or ISRE luciferase reporter (firefly luciferase; $100 \mathrm{ng}$ ) and pRL-TK (renilla luciferase plasmid; $10 \mathrm{ng}$ ) together with $100 \mathrm{ng}$ plasmid encoding Flag-RIG-I, Flag-RIG-I (N), Flag-MDA5, Flag-MDA5 (N), Flag-MAVS, FlagTRIF, Flag-TBK1 or Flag-IRF3, and increasing concentrations ( 0 , 200 , or $400 \mathrm{ng}$ ) of plasmid expressing USP3 or $400 \mathrm{ng}$ plasmid expressing USP3 containing the $\mathrm{ZnF}$ or UCH. Empty pcDNA3.1 vector was used to maintain equal amounts of DNA among wells. Cells were collected at 24-36 h after transfection and luciferase activity was measured with a Dual-Luciferase Assay (Promega) with a Luminoskan Ascent luminometer (Thermo Scientific) according to the manufacturer's protocol. Reporter gene activity was determined by normalization of the firefly luciferase activity to renilla luciferase activity. An Amaxa nucleofector kit $\mathrm{V}$ was used according to the manufacturer's protocols (Lonza Amaxa) for transfection of plasmids or siRNAs into THP-1 cells.

\section{Immunoprecipitation and immunoblot analysis}

For immunoprecipitation, whole-cell extracts were prepared after transfection or stimulation with appropriate ligands, followed by incubation overnight with the appropriate antibodies plus Protein $\mathrm{A} / \mathrm{G}$ beads (Pierce). For immunoprecipitation with anti-Flag or anti-hemagglutinin, anti-Flag or anti-hemagglutinin agarose gels (Sigma) were used. Beads were then washed five times with low-salt lysis buffer, and immunoprecipitates were eluted with $3 \times$ SDS Loading Buffer (Cell Signaling Technology) and resolved by SDS-PAGE. Proteins were transferred to nitrocellulose membranes (Bio-Rad) and further incubated with the appropriate antibodies. LumiGlo Chemiluminescent Substrate System (KPL) was used for protein detection.

\section{In vitro deubiquitination assay}

Ubiquitinated RIG-I or MDA5 was isolated from $293 \mathrm{~T}$ cells transfected with expression vectors for HA-Ub and Flag-RIG-I or Flag-MDA5, and treated with intracellular Poly(I:C). Ubiquitinated RIG-I or MDA5 was purified from the cell extracts with anti-Flag affinity column in Flag-lysis buffer $(50 \mathrm{mM}$ Tris- $\mathrm{HCl}(\mathrm{pH}$ 7.8), $137 \mathrm{mM} \mathrm{NaCl}, 10 \mathrm{mM} \mathrm{NaF}, 1 \mathrm{mM}$ EDTA, $1 \%$ Triton X-100, $0.2 \%$ Sarcosyl, $1 \mathrm{mM}$ DTT, $10 \%$ glycerol and fresh proteinase inhibitors). After extensive washing with the Flag-lysis buffer, the proteins were eluted with Flag peptides (Sigma). The recombinant Flag-USP3 and its mutants were expressed in 293T cells and purified using Flag affinity column and eluted with Flag peptide. For in vitro deubiquitination assay, ubiquitinated RIG-I or MDA5 protein was incubated with recombinant USP3 or its mutants in the deubiquitination buffer (50 mM Tris- $\mathrm{HCl}(\mathrm{pH} 8.0), 50 \mathrm{mM} \mathrm{NaCl}$, $1 \mathrm{mM}$ EDTA, $10 \mathrm{mM}$ DTT, $5 \%$ glycerol) for $2 \mathrm{~h}$ at $37^{\circ} \mathrm{C}$ and then immunoprecipitated with anti-RIG-I or anti-MDA5 plus Protein A/ $\mathrm{G}$ beads (Pierce) for immunoblot analysis.

\section{Cytokine release assay}

Human IFN- $\beta$ was detected with ELISA kits according to the manufacturer's protocols (PBL Biomedical Laboratories).

\section{Virus infection}

VSV-eGFP was provided by S Balachandran. Cells were infected at various MOI as described [25].

\section{Knockdown of USP3 by RNA interference}

Human USP3-specific (HSS115094), mouse USP3-specific (MSS215066) and control (2-scramble mix) siRNA oligonucleotides were obtained from Invitrogen and Integrated DNA Technologies (IDT). Three USP3-specific shRNA plasmids (USP3 human GIPZ Lentiviral shRNA, Clone ID: V2LHS 12524, V2LHS_197713 and V3LHS_320140) and control shRNA plasmids were obtained from Openbiosystems. They were transfected into 293T, THP-1 cells or Raw264.7 cells using Lipofectamine 2000 (Invitrogen) or Nucleofector kit $\mathrm{V}$ according to the manufacturer's instruction.

\section{Statistical analysis}

Data are represented as mean \pm SD when indicated, and Student's $t$-test was used for all statistical analyses with the GraphPad Prism 4.0 software. Differences between groups were considered significant when $P$ value was less than 0.05 .

\section{Acknowledgments}

We would like to thank Dr Jianhua Yang (Baylor College of Medicine) for USP3 and other USP (without tag) plasmids. This work was in part supported by grants (CA090327, CA101795, CA121191, CA116408, CA094327 and DA030338) from NCI, NIH and Cancer Prevention and Research Institute of Texas (CPRIT RP121048) (to RFW), and the National Natural Science Foundation of China (31000394 and 31370869), the National Key Basic Research Program of China (2014CB910800), Guangdong Innovative Research Team Program (2011Y035 and 201001Y0104687244) and Guangdong Natural Science Funds for Distinguished Young Scholar (S2013050014772). 


\section{References}

1 Takeuchi O, Akira S. Pattern recognition receptors and inflammation. Cell 2010; 140:805-820.

2 Loo YM, Gale M Jr. Immune signaling by RIG-I-like receptors. Immunity 2011; 34:680-692.

3 Barbalat R, Ewald SE, Mouchess ML, Barton GM. Nucleic acid recognition by the innate immune system. Annu Rev Immunol 2011; 29:185-214.

4 Chiu YH, Macmillan JB, Chen ZJ. RNA polymerase III detects cytosolic DNA and induces type I interferons through the RIG-I pathway. Cell 2009; 138:576-591.

5 Ablasser A, Bauernfeind F, Hartmann G, Latz E, Fitzgerald KA, Hornung V. RIG-I-dependent sensing of poly(dA:dT) through the induction of an RNA polymerase III-transcribed RNA intermediate. Nat Immunol 2009; 10:1065-1072.

6 Takaoka A, Wang Z, Choi MK, et al. DAI (DLM-1/ZBP1) is a cytosolic DNA sensor and an activator of innate immune response. Nature 2007; 448:501-505.

7 Fernandes-Alnemri T, Yu JW, Datta P, Wu J, Alnemri ES. AIM2 activates the inflammasome and cell death in response to cytoplasmic DNA. Nature 2009; 458:509-513.

8 Hornung V, Ablasser A, Charrel-Dennis M, et al. AIM2 recognizes cytosolic dsDNA and forms a caspase-1-activating inflammasome with ASC. Nature 2009; 458:514-518.

9 Unterholzner L, Keating SE, Baran M, et al. IFI16 is an innate immune sensor for intracellular DNA. Nat Immunol 2010; 11:997-1004.

10 Zhang Z, Yuan B, Bao M, Lu N, Kim T, Liu YJ. The helicase DDX41 senses intracellular DNA mediated by the adaptor STING in dendritic cells. Nat Immunol 2011; 12:959-965.

11 Theofilopoulos AN, Baccala R, Beutler B, Kono DH. Type I interferons (alpha/beta) in immunity and autoimmunity. Annu Rev Immunol 2005; 23:307-336.

12 Eisenacher K, Krug A. Regulation of RLR-mediated innate immune signaling -- it is all about keeping the balance. Eur $J$ Cell Biol 2012; 91:36-47.

13 Liew FY, Xu D, Brint EK, O’Neill LA. Negative regulation of toll-like receptor-mediated immune responses. Nat Rev Immunol 2005; 5:446-458.

14 Bhoj VG, Chen ZJ. Ubiquitylation in innate and adaptive immunity. Nature 2009; 458:430-437.

15 Peisley A, Lin C, Wu B, et al. Cooperative assembly and dynamic disassembly of MDA5 filaments for viral dsRNA recognition. Proc Natl Acad Sci USA 2011; 108:21010-21015.

16 Peisley A, Jo MH, Lin C, et al. Kinetic mechanism for viral dsRNA length discrimination by MDA5 filaments. Proc Natl Acad Sci USA 2012; 109:E3340-E3349.

17 Hou F, Sun L, Zheng H, Skaug B, Jiang QX, Chen ZJ. MAVS forms functional prion-like aggregates to activate and propagate antiviral innate immune response. Cell 2011; 146:448461.

18 Jiang F, Ramanathan A, Miller MT, et al. Structural basis of RNA recognition and activation by innate immune receptor RIG-I. Nature 2011; 479:423-427.

19 Kowalinski E, Lunardi T, McCarthy AA, et al. Structural basis for the activation of innate immune pattern-recognition receptor RIG-I by viral RNA. Cell 2011; 147:423-435.

20 Zeng W, Sun L, Jiang X, et al. Reconstitution of the RIG-I pathway reveals a signaling role of unanchored polyubiquitin chains in innate immunity. Cell 2010; 141:315-330.

21 Gack MU, Shin YC, Joo CH, et al. TRIM25 RING-finger E3 ubiquitin ligase is essential for RIG-I-mediated antiviral activity. Nature 2007; 446:916-920.

22 Oshiumi H, Miyashita M, Inoue N, Okabe M, Matsumoto $\mathrm{M}$, Seya T. The ubiquitin ligase riplet is essential for RIG-Idependent innate immune responses to RNA virus infection. Cell Host Microbe 2010; 8:496-509.

23 Nicassio F, Corrado N, Vissers JH, et al. Human USP3 is a chromatin modifier required for $\mathrm{S}$ phase progression and genome stability. Curr Biol 2007; 17:1972-1977.

24 Karin M, Lawrence T, Nizet V. Innate immunity gone awry: linking microbial infections to chronic inflammation and cancer. Cell 2006; 124:823-835.

25 Cui J, Zhu L, Xia X, et al. NLRC5 negatively regulates the NF-kappaB and type I interferon signaling pathways. Cell 2010; 141:483-496.

26 Xia X, Cui J, Wang HY, et al. NLRX1 negatively regulates TLR-induced NF- $\kappa$ B signaling by targeting TRAF6 and IKK. Immunity 2011; 34:843-853.

27 Moore CB, Bergstralh DT, Duncan JA, et al. NLRX1 is a regulator of mitochondrial antiviral immunity. Nature 2008; 451:573-577.

28 Allen IC, Moore CB, Schneider M, et al. NLRX1 protein attenuates inflammatory responses to infection by interfering with the RIG-I-MAVS and TRAF6-NF-kappaB signaling pathways. Immunity 2011; 34:854-865.

29 Jia Y, Song T, Wei C, et al. Negative regulation of MAVSmediated innate immune response by PSMA7. J Immunol 2009; 183:4241-4248.

30 You F, Sun H, Zhou X, et al. PCBP2 mediates degradation of the adaptor MAVS via the HECT ubiquitin ligase AIP4. Nat Immunol 2009; 10:1300-1308.

31 Cui J, Li Y, Zhu L, et al. NLRP4 negatively regulates type I interferon signaling by targeting the kinase TBK1 for degradation via the ubiquitin ligase DTX4. Nat Immunol 2012; 13:387-395.

32 Luo D, Ding SC, Vela A, Kohlway A, Lindenbach BD, Pyle AM. Structural insights into RNA recognition by RIG-I. Cell 2011; 147:409-422.

33 Oshiumi H, Matsumoto M, Hatakeyama S, Seya T. Riplet/ RNF135, a RING finger protein, ubiquitinates RIG-I to promote interferon-beta induction during the early phase of viral infection. J Biol Chem 2009; 284:807-817.

34 Jiang X, Kinch LN, Brautigam CA, et al. Ubiquitin-induced oligomerization of the RNA sensors RIG-I and MDA5 activates antiviral innate immune response. Immunity 2012; 36:959-973.

35 Lin R, Yang L, Nakhaei P, et al. Negative regulation of the retinoic acid-inducible gene I-induced antiviral state by the ubiquitin-editing protein A20. J Biol Chem 2006; 281:20952103.

36 Zhang M, Wu X, Lee AJ, et al. Regulation of IkappaB kinaserelated kinases and antiviral responses by tumor suppressor CYLD. J Biol Chem 2008; 283:18621-18626.

37 Arimoto K, Takahashi H, Hishiki T, Konishi H, Fujita T, Shimotohno K. Negative regulation of the RIG-I signaling by the ubiquitin ligase RNF125. Proc Natl Acad Sci USA 2007; 
104:7500-7505.

38 Chen W, Han C, Xie B, et al. Induction of Siglec-G by RNA viruses inhibits the innate immune response by promoting RIG-I degradation. Cell 2013; 152:467-478.
39 Chen R, Zhang L, Zhong B, Tan B, Liu Y, Shu HB. The ubiquitin-specific protease 17 is involved in virus-triggered type I IFN signaling. Cell Res 2010; 20:802-811.

(Supplementary information is linked to the online version of the paper on the Cell Research website.) 